\title{
Stokes Flow in Thin Films
}

Present understanding of the mechanisms of lubrication and the load carrying capacity of lubricant films mainly relies on models in which the Reynolds equation is used to describe the flow. The narrow gap assumption is a key element in its derivation from the Navier Stokes equations. However, the tendency in applications is that lubricated contacts have to operate at smaller film thickness levels, and because engineering surfaces are never perfectly smooth, locally in the film this narrow gap assumption may violated. In addition to this geometric limitation of the validity of the Reynolds equation may come a piezoviscous and compressibility related limitation. In this paper the accuracy of the predictions of the Reynolds model in relation to the local geometry of the gap is investigated. A numerical solution algorithm for the flow in a narrow gap has been developed based on the Stokes equations. For a model problem the differences between the pressure and velocity fields according to the Stokes model and the Reynolds equation have been investigated. The configuration entails a lower flat surface together with an upper surface (flat or parabolic) in which a local defect (single asperity) of known geometry has been embedded. It is investigated how the magnitude of the differences develops as a function of the geometric parameters of the film and the feature. Finally, it is discussed to what extend for these problems a perturbation approach can provide accurate corrections to be applied to the Reynolds solution. [DOI: 10.1115/1.1506317]

\section{Introduction}

Technical surfaces are never perfectly smooth and an important question is how to account for the effect of the surface roughness on the film formation. This has been the subject of many studies in the past decades. Clearly if the surfaces are so smooth that the computed minimum film thickness for the given operating conditions is large compared to the standard deviation of the surface roughness profile in the contact the effect of the roughness will be negligible. However, if the amplitude of the roughness is comparable to the thickness of the film one has to take into account its effect on the film.

For a Newtonian fluid the Navier-Stokes (NS) equations describe the conservation of momentum. If the inertia terms are small compared to the viscous terms, they reduce to the Stokes equations. Assuming next that $H / L \ll 1$, where $H$ is the local film thickness and $L$ some typical length scale in the contact it then follows from the Stokes equations that the pressure will be independent of the cross-film coordinate which facilitates integration over the cross-film coordinate to obtain the velocity field and eventually leads to the Reynolds [1] equation from which the pressure can be solved. However, for the case of non-smooth surfaces the assumption $H / L \ll 1$ may (locally) be violated. This is already suggested by Elrod [2] where a table of values of a ratio $H / \Lambda_{c}$ is presented as can appear in applications where $H$ is the film thickness and $\Lambda_{c}$ a characteristic roughness wavelength. It is argued that this ratio could be as large as $O(1)$.

Based on the anticipated validity of the Reynolds equation roughness has been classified in two categories: Reynolds roughness and Stokes roughness, see Elrod [9]:

- Reynolds roughness $H / \Lambda_{c} \ll 1$.

- Stokes roughness $H / \Lambda_{c}=O(1)$.

Reynolds roughness implies it is assumed that it is justified to use the Reynolds equation to describe the fluid flow in the lubricated contact, otherwise the Stokes equations should be used. It is not clear where the border between Reynolds and Stokes roughness should be drawn and how it is related to the problem parameters.

A second classification of roughness effect studies that has been made is based on the way in which the roughness effects are

Contributed by the Tribology Division for publication in the ASME JOURNAL OF TRIBOLOGY. Manuscript received by the Tribology Division October 19, 2001 revised manuscript received June 26, 2002; Associate Editor: L. San Andrés. incorporated in a model (either Stokes or Reynolds). Two approaches are distinguished: a deterministic approach and a statistical approach. The statistical approach is based on the characterization of a surface roughness profile by means of statistical parameters. "Statistical" model equations are derived, e.g., a generalized Reynolds equation, in which the coefficients are a function of various statistical properties of the roughness. The most common quantities appearing in such models are the Root Mean Square (RMS) value and the auto-correlation length. In general it has been observed that the rough surface analysis yields a larger load capacity then obtained for the smooth surface case. Examples of this approach based on the Reynolds equation are the works of Tzeng and Saibel $[3,4]$, Christensen and co-workers [5-8], Elrod $[9,10]$, Cheng and Dyson [11] and also Chow and Cheng [12], Chow and Saibel [13], Sun [14], Phan Thien [15-17], Phan-Thien and Atkinson, [18] and Prakash [19]. A related approach referred to as "the flow factor method" is where the details of the roughness enter in coefficients of the Reynolds equation as so-called flow factors, see Patir and Cheng [20, 21], Teale and Lebeck [22], Elrod [2], and Tripp [23]. This flow factor method is widely used in practice in both hydrodynamic lubrication as well as ElastoHydrodynamic Lubrication (EHL) problems. Examples of the latter are found in the works of Sadeghi and Sui [24], Patir and Cheng [25], and Tripp and Hamrock [26].

The deterministic approach differs from the statistical approach in that the model equations, Reynolds or Stokes, are solved taking for the film thickness an exact description of the surface geometry as a function of the coordinates. An early example of deterministic studies in the field of EHL are the papers of Goglia et al. [27,28]. In general the model equations have to be solved numerically. An accurate description of small scale surface features requires many gridpoints so, for a deterministic approach to be feasible, fast computers and, more important, efficient numerical algorithms, are essential. This is clearly illustrated by the developments in the field of EHL modeled using Reynolds' equation. Fast and stable algorithms based on multigrid/multilevel techniques aided by the developments in terms of faster computers have led to a situation where at present the effects of surface features on film formation in EHL point contacts can be simulated using a deterministic approach, even taking into account the transient effects of the surface feature moving through the contact. Examples of such studies are the papers of Venner et al. [29,30] and Wijnant et al. [31]. At the same time on the experimental side the optical interferometry 
technique to measure the film thickness in model contacts improved so much that it has become possible to validate predicted film thickness behavior even on a sub-contact scale. This is for example illustrated by the work of Kaneta [32]. The agreement between the computed film shapes and the measured profiles is good, as was for example shown by Venner and Lubrecht [33], for the particular case of an artificial ridge moving through the contact. However, also some local discrepancies were observed that could not be explained. Venner and Lubrecht [33] suggest that these differences could be an indication of limitations to the (local) validity of the Reynolds equation.

Most likely because of the additional dimension and the associated complications related to the analysis and numerical solution only few studies exist in which the Stokes problem or Stokes roughness is considered. Some of the earliest examples are the works of Sun and Chen [34] and of a later date the paper of Phan-Thien [35] using the statistical approach. Stokes roughness effects were also the subject of the works of Myllerup and Hamrock $[36,37]$ but here a perturbation approach was used rather than the full Stokes equations. Only a few studies have appeared in which a lubrication (related) problem described with the Stokes equations is solved numerically. One example is the work of Noordmans [38] where an algorithm was developed to solve the Stokes equations on a highly anisotropic-rectangular domain. Schäfer et al. [39] solved the EHL problem but they used modified Stokes equations in which some viscous terms were omitted to describe the fluid flow. They showed that especially when sliding occurs the Reynolds solution differs significantly from the solution of their modified Stokes equations. Finally Almqvist [40] recently presented results obtained for an EHL line contact problem using the Navier-Stokes equations solved with a commercial computational fluid dynamics (CFD) package. Only a few results were presented in which differences between the Reynolds and Navier-Stokes results were reported. It appears that the computations were hampered by stability and computing time problems. However, one interesting result is that for a model problem of a converging gap an illustration is given of what can be referred to as the piezoviscous limitation to the validity of the Reynolds equation that was suggested by Bair et al. [41].

In conclusion, the limits for which the solutions to the Reynolds equation are accurate should be further explored. In this paper solutions obtained with the Reynolds model, a corrected Reynolds model, and a Stokes model for a thin film flow problem are compared. For this problem the geometric limitation to the Reynolds solutions is investigated. In particular the errors in the flow field quantities in the Reynolds and corrected Reynolds solutions are studied in relation to the (local) film aspect ratio $H / L$.

\section{Theory}

The Stokes equations follow from the Navier-Stokes equations after omitting the inertia terms (slow viscous flow) and assuming an incompressible isoviscous fluid. Although in a thin film thermal effects can play an important role, in this study isothermal conditions are assumed. The objective is to really isolate phenomena induced by the geometry. Furthermore, only two-dimensional, steady flow problems are studied. Below first the Stokes equations are presented. Subsequently a perturbation analysis is presented in terms of the film aspect ratio $\epsilon=H / L$. The limiting case of $\epsilon$ $=0$ leads to the well known Reynolds equation. Also expressions for the first and second order corrections to the Reynolds solution are presented.

To analyze the equations dimensionless variables have been introduced as suggested by Myllerup et al. [37]. Assuming a characteristic speed $u_{c}$, a characteristic length $L$ in the $x$-direction, a characteristic height $H$ in the $z$-direction, and a characteristic viscosity $\eta_{c}$ the variables can be transformed according to:

$$
\begin{gathered}
u \rightarrow u_{c} u, \\
w \rightarrow \epsilon u_{c} w, \quad \epsilon=\frac{H}{L}, \\
x \rightarrow L x, \\
z \rightarrow H z, \\
p \rightarrow \frac{\eta_{c} u_{c} L}{H^{2}} p .
\end{gathered}
$$

In terms of these dimensionless variables the Stokes equations are given by:

$$
\begin{gathered}
\frac{\partial p}{\partial x}-\epsilon^{2} \frac{\partial^{2} u}{\partial x^{2}}-\frac{\partial^{2} u}{\partial z^{2}}=0, \\
\frac{\partial p}{\partial z}-\epsilon^{4} \frac{\partial^{2} w}{\partial x^{2}}-\epsilon^{2} \frac{\partial^{2} w}{\partial z^{2}}=0, \\
\frac{\partial u}{\partial x}+\frac{\partial w}{\partial z}=0 .
\end{gathered}
$$

The system formed by Eqs. (2), (3), and (4) is of elliptic type and will henceforth be referred to as the "Stokes model."

For $\epsilon=O(1)$ all terms in the equations are of equal importance for the solution so the full system must be solved. In lubrication problems $\epsilon$ is usually small. As will be shown below, for the limit of negligibly small $\epsilon$ one can simplify the problem to a single equation: The Reynolds equation. The solutions to this equation will be accurate approximations to the solutions of the full problem as long as $\epsilon$ is small. For an intermediate regime of values equations can be derived for corrections to the Reynolds solution by means of a perturbation approach. The advantage of such corrections is that they are generally still easier to obtain than solutions to the full Stokes model.

A regular expansion in $\epsilon$ for the dependent variables in Eqs. (2), (3), and (4) was used, as proposed by Myllerup et al. [37]. The perturbation parameter chosen must be small to give a convergent expansion. Introducing the parameter $\gamma=\epsilon^{2}$, the dependent variables can be expanded in the following way:

$$
\begin{gathered}
u(x, z)=u_{0}(x)+\gamma u_{1}(x, z)+\gamma^{2} u_{2}(x, z)+O\left(\gamma^{3}\right), \\
w(x, z)=w_{0}(x, z)+\gamma w_{1}(x, z)+\gamma^{2} w_{2}(x, z)+O\left(\gamma^{3}\right), \\
p(x, z)=p_{0}(x)+\gamma p_{1}(x, z)+\gamma^{2} p_{2}(x, z)+O\left(\gamma^{3}\right) .
\end{gathered}
$$

Substitution of Eq. (5) in the Eqs. (2)-(4) and equating equal orders in $\gamma$ gives for the zero order:

$$
\begin{gathered}
\frac{\partial p_{0}}{\partial x}-\frac{\partial^{2} u_{0}}{\partial z^{2}}=0, \\
\frac{\partial p_{0}}{\partial z}=0, \\
\frac{\partial u_{0}}{\partial x}+\frac{\partial w_{0}}{\partial z}=0 .
\end{gathered}
$$

The velocity $u_{0}$ follows from integration of Eq. (6) twice with respect to $z$, using the boundary conditions. Defining $z=0$ to be the lower surface and $z=h(x)$ the upper surface the boundary conditions will be $u_{0}(x, 0)=U_{0}$ and $u_{0}(x, h(x))=U_{h}$ giving:

$$
u_{0}=\frac{1}{2} \frac{d p_{0}}{d x} z(z-h)+\left(U_{h}-U_{0}\right) \frac{z}{h}+U_{0} .
$$

Substitution of this result in Eq. (8) followed by integration with respect to $z$, using "Leibniz" rule yields the Reynolds equation for $p_{0}(x)$ : 


$$
\frac{d}{d x}\left[6\left(U_{h}+U_{0}\right) h(x)-h^{3}(x) \frac{d p_{0}}{d x}\right]=0 .
$$

Finally an expression for $w_{0}$ can be deduced from Eq. (8) and Eq. (9):

$$
w_{0}=\frac{z^{2}(z-h) \frac{d h}{d x}\left(h^{2} \frac{d p_{0}}{d x}+2\left(U_{h}-U_{0}\right)\right)}{2 h^{3}} .
$$

In this paper the problems considered will be restricted to a flat lower surface with speed $u_{c}$ which gives $u_{0}(x, 0)=1$ and a stationairy upper surface $u_{0}(x, h)=0$.

Clearly, the zero order terms $u_{0}, w_{0}$, and $p_{0}$, form the Reynolds solution to the problem. The same approach can now be followed to find the first and second order expansion terms, see Appendix A. For example, up to second order the pressure is given by:

$$
\begin{aligned}
p(x, z)= & p_{0}(x)+\gamma \underbrace{\left(\tilde{p}_{1}(x)+\frac{\partial w_{0}}{\partial z}\right)}_{p_{1}(x, z)} \\
& +\gamma^{2}(\underbrace{\left.\int\left(\frac{\partial^{2} w_{0}}{\partial x^{2}}+\frac{\partial^{2} w_{1}}{\partial z^{2}}\right) d z+\tilde{p}_{2}(x)\right)}_{p_{2}(x, z)},
\end{aligned}
$$

In appendix $A$ the expressions for $\widetilde{p}_{1}(x)$ and $\widetilde{p}_{2}(x)$ are given. It is expected that for small but not very small $\epsilon$ corrections to $u_{0}, w_{0}$, and $p_{0}$ obtained in this way would yield improved solutions. However, it is noted that even for small $\epsilon$ the expansion can break down in regions of large gradients in $h(x)$. For such cases a more sophisticated perturbation. should be used probably based on a combination of expansion parameters, i.e., $\epsilon$ on a global scale and some $H_{\mathrm{loc}} / \Lambda_{c}$ on a local scale where $H_{\mathrm{loc}}$ and $\Lambda_{c}$ are a characteristic film thickness and wavelength in the large gradient region.

\section{Model Problem}

Solutions to the pressure and velocities according to the Stokes model, the Reynolds equation and to the perturbation equations of first and second order will be compared for a model problem. Figure 1 shows a typical configuration. Let the gap between the surfaces in dimensionless variables be given by the function:

$$
h(x)=\left(1-h_{\mathrm{loc}}\right) x^{n_{s}}+h_{\mathrm{loc}}-R^{\prime}(x),
$$

for $-1 \leqslant x \leqslant 1$. The first two terms represents the "global" shape of the channel. The third term represents a local feature in the film. In this paper two cases will be considered. First $n_{s}=0$ which is a uniform channel with height $h_{\text {loc }}$. Secondly $n_{s}=4$ which gives an approximation of a typical EHL film shape: A "para-

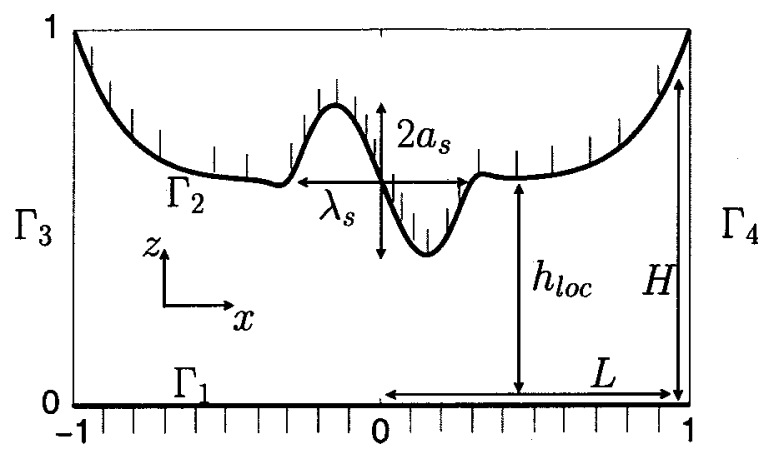

Fig. 1 Geometry for single local feature bolic" type channel with a flat region in the center of thickness $h_{\text {loc }}$. In both cases for the local feature the following function is used:

$$
R^{\prime}(x)=a_{s}^{\prime} \frac{\sin \left(2 \pi x / \lambda_{s}\right)}{\left(\left(1+e^{-\kappa_{s}\left(\Lambda_{s} / 2-x\right)}\right)\left(1+e^{-\kappa_{s}\left(\Lambda_{s} / 2+x\right)}\right)\right)},
$$

where

$$
a_{s}^{\prime}=a_{s}\left(1+e^{-\kappa_{s}\left(\Lambda_{s} / 2-\lambda_{s} / 4\right)}\right)\left(1+e^{-\kappa_{s}\left(\Lambda_{s} / 2+\lambda_{s} / 4\right)}\right) .
$$

Eq. (14) and Eq. (15) represent a "wavetrain" of width $\Lambda_{s}$ and the wavelength of the oscillation $\lambda_{s} . \kappa_{s}$ is a decay parameter. In the limit of large $\kappa_{s}$ there are $\Lambda_{s} /\left(2 \lambda_{s}\right)$ wavelengths in the feature on each side. In a uniform channel the maximum value $h=h_{\text {loc }}+a_{s}$ is attained at $x=-\lambda_{s} / 4$ and the minimum value $h=h_{\mathrm{loc}}-a_{s}$ at $x$ $=\lambda_{s} / 4$. In this study $\kappa_{s}$ will be fixed $\kappa_{s}=16 / \lambda_{s}$ and $\Lambda_{s}=\lambda_{s}$ so one obtains a single wavelength which can be seen as a combination of a valley and an asperity. With respect to the amplitude for $a_{s}=h_{\text {loc }}$ the asperity completely blocks the channel. In Fig. 1 the configuration for $n_{s}=4, h_{\mathrm{loc}}=0.6, a_{s}=0.2$, and $\lambda_{s}=0.6$ is shown.

It is noted that $a_{s}$ and $\lambda_{s}$ are dimensionless parameters. In physical terms the amplitude of the feature is $A=H a_{s}$ with $H$ the nominal film thickness and its wavelength is $\Lambda=L \lambda_{s}$ with $L$ the nominal length of the domain.

In Section 5.1 results will be presented for a single feature in a uniform channel of unit height varying $a_{s}, \lambda_{s}$ and the parameter $\epsilon=H / L$ that appears in the Stokes equations. In Section 5.3 the results for a local feature in a channel with an EHL film type of geometry are presented. For this case $h_{\mathrm{loc}}, \lambda_{s}$, and $a_{s}$ were varied for different values of $\epsilon$. The ranges of values considered for each parameter are given in the relevant sections.

In Fig. 1 the boundaries of the domain are indicated with $\Gamma_{1}, \ldots, \Gamma_{4}$. The boundary conditions for the flow in the gap will be given in terms of the velocity of the surfaces and the pressure at the inlet and outlet of the film:

$$
\begin{array}{ll}
\Gamma_{1}: u=1, & w=0, \\
\Gamma_{2}: u=0, & w=0, \\
\Gamma_{3}: p=0, & w=0, \\
\Gamma_{4}: p=0, & w=0 .
\end{array}
$$

The pressure on the boundaries $\Gamma_{3}$ and $\Gamma_{4}$ is taken zero as usually the pressure will be large compared to the ambient pressure.

In this article only steady flow situations are considered so the surface on which a feature is present $\left(\Gamma_{2}\right.$ in Fig. 1) is assumed to be stationary. The difference between solutions obtained for the problem with the various models (Stokes, Reynolds, corrected Reynolds) will be shown in terms of the flow field variables pressure and velocity and by means of the generated load carrying capacity (lift force). In most tribological problems a large pressure rise occurs in a region of narrowing gap. However, an equally large pressure fall will be predicted in a widening region. For a symmetric geometry this implies that the predicted load that could be carried is zero. However, in practice a lubricant (fluid) can not sustain negative pressures and in the widening region the pressure drops to the vapor pressure (usually taken equal to the ambient pressure). As a result the net load capacity is nonzero. To simulate this physical cavitation behavior the model should be extended with a cavitation condition. This can be done by means of the density pressure relation. However, as the compressibility behavior itself can cause differences between Reynolds and Stokes solutions, here, to separate the issues, a simplified approach is used and the load capacity is computed from a given solution in the "Sommerfeld" manner: Only the positive pressure values are used and the negative pressure values in the computed flow field are replaced by zero (or sub-vapor pressures by the vapor pressure). So, for the computation of the load capacity the pressure field is cut off from below at zero. Compared with the pressure field that would be obtained if a cavitation condition were imposed this 
truncated field only differs near the cavitation boundary where the pressure gradient would be zero which it obviously is not in the truncated Sommerfeld field. However, the error that is made in the computed load capacity is small because the pressures near the cavitation boundary in both cases are small and contribute little to the integral over the field.

Summarizing, the difference between the Stokes and the Reynolds solution has been measured by means of the generated load capacity which was defined as:

$$
\bar{R}=\frac{\int \tilde{\Gamma}_{1} p_{S}(x, z=0) d x-\int \tilde{\Gamma}_{1} p_{R}(x) d x}{\int \tilde{\Gamma}_{1} p_{R}(x) d x},
$$

where the tilde in $\widetilde{\Gamma}$ indicates that the pressure is only integrated for positive values. The effect of this approximation on the behavior of $\bar{R}$ as a function of the geometry and the film aspect ratio is shown to be small in [42].

Note that for the relative difference between the Stokes and the Reynolds solution it makes little difference if the lower or the upper surface is taken in Eq. 17. However, given that the lower surface is assumed to be flat in the model problems considered the calculation using $p(x, z=0)$ is simply easier. Which surface is taken is not an issue for the load. It would be an issue for the friction force.

\section{Numerical Solution}

In the Reynolds equation the shape of the gap is no issue in the discretization of the equation for numerical solution and the implementation of the boundary conditions. It appears in the equations as a (known) function $h(x)$ and the boundary conditions have been taken care of when integrating the momentum equation over the film. This is not the case for the Stokes equations. To easily deal with the non-rectangular boundaries and the implementation of the boundary conditions the problem has been transformed to a rectangular domain using curvi-linear coordinates. On the transformed domain the equations have been discretized. For the numerical solution of the resulting system of discrete equations Multigrid (MG) techniques have been used. In this section details of the transformation, the discretization, and the numerical solution procedure are given.

4.1 Curvi-linear Coordinates. The equations are transformed from the physical domain to a rectangular computational domain and the transformed equations are then discretized on this rectangular domain as was done by Oosterlee [44]. In our tribological context the flow domain is usually rather straightforward, i.e. no flow around a corner. This implies that generally no steep gradients in the grid lines will occur, i.e., while $\epsilon$ may not be very small, it will still not be large. As a result it is sufficient to transform only the $x$ and $z$-coordinate and there is no need to transform the velocity vector too. This has the advantage that the task of discretizing the Christoffel symbols [45] which would result from the velocity transformation, does not arise.

Let the relation between the physical coordinates $\mathbf{x}=\left(x^{1}, x^{2}\right)$ $=(x, z) \in \Omega$ and the computational coordinates $\left(\xi^{1}, \xi^{2}\right) \in G$, with $G$ the rectangular computational domain, be given by $\mathbf{x}$ $=\mathbf{x}\left(\xi^{1}, \xi^{2}\right)$. The inverse transformation should exist and let $\sqrt{g}$ be the determinant of the Jacobian $J$ of the transformation.

In this article all results are obtained with a transformation of the $x^{2}$ direction using the gap height $h\left(x^{1}\right)$ :

$$
x=x\left(\xi^{1}\right), \quad z=\xi^{2} h\left(\xi^{1}\right) .
$$

Eqs. (2), (3), and (4) transformed to curvilinear coordinates in a conservative form can be written as:

$$
\begin{gathered}
\epsilon^{2} \frac{\partial}{\partial \xi^{k}}\left[\sqrt{g} a_{1}^{(k)} a_{1}^{(l)} \frac{\partial u}{\partial \xi^{l}}\right]+\frac{\partial}{\partial \xi^{k}}\left[\sqrt{g} a_{2}^{(k)} a_{2}^{(l)} \frac{\partial u}{\partial \xi^{l}}\right]=\epsilon \frac{\partial}{\partial \xi^{k}}\left(\sqrt{g} a_{1}^{(k)} p\right), \\
\epsilon^{3} \frac{\partial}{\partial \xi^{k}}\left[\sqrt{g} a_{1}^{(k)} a_{1}^{(l)} \frac{\partial w}{\partial \xi^{l}}\right]+\epsilon \frac{\partial}{\partial \xi^{k}}\left[\sqrt{g} a_{2}^{(k)} a_{2}^{(l)} \frac{\partial w}{\partial \xi^{l}}\right]=\frac{\partial}{\partial \xi^{k}}\left(\sqrt{g} a_{2}^{(k)} p\right), \\
\frac{\partial}{\partial \xi^{k}}\left(\sqrt{g} a_{l}^{(k)} u^{l}\right)=0 .
\end{gathered}
$$

where $a_{l}^{(k)}$ are the contravariant base vectors which are the vectors normal to the $\xi^{k}=$ constant lines. In practice, because the transformation is given in the form $\mathbf{x}=\mathbf{x}\left(\xi^{1}, \xi^{2}\right)$, it is convenient to replace them in the equations by expressions in terms of the covariant base vectors which follow from the fact that the two types of base vectors form an orthonormal set, see Nomenclature.

Summarizing, the problem of solving $u, w$, and $p$ as a function of $x$ and $z$ in the domain $\Omega$ from Eqs. (2), (3), and (4) is now replaced by solving $u, w, p$ as a function of $\xi_{1}$ and $\xi_{2}$ in the domain $G$ from Eqs. (19), (20), and (21).

4.2 Discretization. The physical domain $\Omega$ is bounded on four sides. In the transformed space $G$ each line with $\xi^{i}=0,1$ corresponds to one of the sides of $\Omega$. The transformed Eqs. (19), (20) and (21) are discretized on a uniform grid. This computational grid is defined by $G_{\delta \xi}=\left\{\left(\xi_{i-1 / 2}^{1}, \xi_{j-1 / 2}^{2}\right)=\left((i-1) \delta \xi^{1},(j\right.\right.$ -1) $\left.\left.\delta \xi^{2}\right) ;(i, j)=1, \ldots, N+1 ; \delta \xi^{1}, \delta \xi^{2}=1 / N\right\}$ and the coordinates of the cell centers are $\left(\xi_{i}^{1}, \xi_{j}^{2}\right)=\left((i-1 / 2) \delta \xi^{1},(j\right.$ $\left.\left.-1 / 2) \delta \xi^{2}\right) ;(i, j)=1, \ldots, N\right)$. From the definition of $G_{\delta \xi}$ it is clear that $\left(\delta \xi^{1}=\delta \xi, \delta \xi^{2}=\delta \xi\right)$ but for clarity the notation $\left(\delta \xi^{1}, \delta \xi^{2}\right)$ is used.

A stable discretization is ensured by a staggered grid arrangement of the variables, as shown in Fig. 2. The pressure is defined in the cell center, the $u$-velocity component on the vertical cell face and the $w$-velocity on the horizontal cell face. Integration of the momentum and continuity equations has been carried out over the corresponding finite volumes. In Fig. 2 the finite volume (FV) corresponding to $u_{i-1 / 2 j}$ is shown. For the $x$-momentum, Eq. (19), Gauss' theorem gives:

$$
\int_{\Gamma_{u}}\left[\epsilon \sqrt{g} a_{1}^{(k)} p-\epsilon^{2} \sqrt{g} a_{1}^{(k)} a_{1}^{(l)} \frac{\partial u}{\partial \xi^{l}}-\sqrt{g} a_{2}^{(k)} a_{2}^{(l)} \frac{\partial u}{\partial \xi^{l}}\right] n_{k} d s=0,
$$

where $\Gamma_{u}$ is the boundary of the FV for the $x$-momentum equation and $n_{k}$ the $k^{\text {th }}$ component of the unit normal outward of this FV. Taking the dependent variables constant when integrating over the cell faces, leads to:

$$
\begin{aligned}
\delta \xi^{2}\{ & \left.\epsilon \sqrt{g} a_{1}^{(1)} p\right\}_{i j}-\delta \xi^{2}\left\{\epsilon \sqrt{g} a_{1}^{(1)} p\right\}_{i-1 j}+\delta \xi^{1}\left\{\epsilon \sqrt{g} a_{1}^{(2)} p\right\}_{i-1 / 2 j+1 / 2} \\
& -\delta \xi^{1}\left\{\epsilon \sqrt{g} a_{1}^{(2)} p\right\}_{i-1 / 2 j-1 / 2}-\delta \xi^{2}\left\{\sqrt { g } \left[\epsilon^{2} a_{1}^{(1)} a_{1}^{(l)} \frac{\partial u}{\partial \xi^{l}}\right.\right. \\
& \left.+a_{2}^{(1)} a_{2}^{(l)} \frac{\partial u}{\partial \xi^{l}}\right\}_{i j}+\delta \xi^{2}\left\{\sqrt{g}\left[\epsilon^{2} a_{1}^{(1)} a_{1}^{(l)} \frac{\partial u}{\partial \xi^{l}}+a_{2}^{(1)} a_{2}^{(l)} \frac{\partial u}{\partial \xi^{l}}\right\}_{i-1 j}\right. \\
& -\delta \xi^{1}\left\{\sqrt{g}\left[\epsilon^{2} a_{1}^{(2)} a_{1}^{(l)} \frac{\partial u}{\partial \xi^{l}}+a_{2}^{(2)} a_{2}^{(l)} \frac{\partial u}{\partial \xi^{l}}\right\}_{i-1 / 2 j+1 / 2}\right. \\
+ & \delta \xi^{1}\left\{\sqrt{g}\left[\epsilon^{2} a_{1}^{(2)} a_{1}^{(l)} \frac{\partial u}{\partial \xi^{l}}+a_{2}^{(2)} a_{2}^{(l)} \frac{\partial u}{\partial \xi^{l}}\right\}_{i-1 / 2 j-1 / 2}=0,\right.
\end{aligned}
$$

where $\delta \xi^{1}$ and $\delta \xi^{2}$ are the length of the cell faces in the $\xi^{1}$ - and $\xi^{2}$-direction, respectively. A similar expression can be found for the $z$-momentum equation at $\left(\xi_{i}^{1}, \xi_{j-1 / 2}^{2}\right)$ taking as $\mathrm{FV}$ the box defined by the four points $\left(\xi_{i-1 / 2}^{1}, \xi_{j}^{2}\right),\left(\xi_{i+1 / 2}^{1}, \xi_{j}^{2}\right),\left(\xi_{i-1 / 2}^{1}, \xi_{j-1}^{2}\right)$, and $\left(\xi_{i+1 / 2}^{1}, \xi_{j-1}^{2}\right)$. 


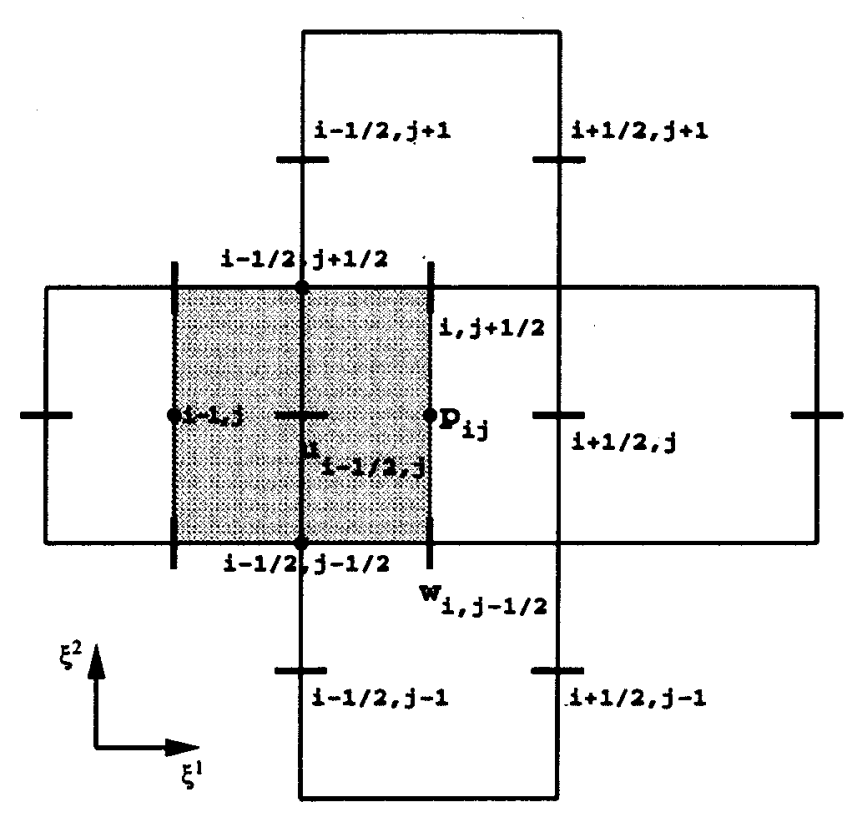

Fig. 2 Illustration of staggered grid cells and numbering and location of variables around a specific gridcell numbered as $(i, j)$. Pressures are defined at cell centers (dot) such as the point $(i, j)$, horizontal velocities at the center of vertical cellfaces (horizontal bar, locations $i-1 / 2, j, i-1 / 2, j+1$, etc.), vertical velocities at the center of horizontal cell faces (vertical bar, locations $i, j-1 / 2, i, j+1 / 2)$. The equation of continuity is defined at pressure points, the $x$ momentum equation at horizontal velocity points, and the $y$ momentum equation at the vertical velocity points. The shaded region indicates the finite volume used the discretization of the $x$-momentum equation in the point $i-1 / 2, j$. If variables are needed at points of this volume at which they are not defined on the grid, e.g., $p_{i-1 / 2, j+1 / 2}$ they are approximated by interpolation, see appendix $B$.

A finite volume for the integration of the continuity equation is centered around $p_{i j}$, see Fig. 2. The integration gives:

$$
\int_{\Gamma_{p}}\left[\sqrt{g} a_{l}^{(k)} u^{l}\right] n_{k} d s=0,
$$

where $\Gamma_{p}$ is the boundary of this $\mathrm{FV}$ for the continuity equation and $n_{k}$ is the $k^{\text {th }}$ component of the unit normal directed outward of the FV. This FV is defined by the four points $\left(\xi_{i-1 / 2}^{1}, \xi_{j-1 / 2}^{2}\right)$, $\left(\xi_{i+1 / 2}^{1}, \xi_{j-1 / 2}^{2}\right),\left(\xi_{i-1 / 2}^{1}, \xi_{j+1 / 2}^{2}\right)$, and $\left(\xi_{i+1 / 2}^{1}, \xi_{j+1 / 2}^{2}\right)$. Taking the dependent variables constant over the cell faces, the integration of Eq. (24) gives:

$$
\begin{aligned}
& {\left[\sqrt{g} a_{l}^{(1)} u^{l}\right]_{i+1 / 2 j} \delta \xi^{2}-\left[\sqrt{g} a_{l}^{(1)} u^{l}\right]_{i-1 / 2 j} \delta \xi^{2}+\left[\sqrt{g} a_{l}^{(2)} u^{l}\right]_{i j+1 / 2} \delta \xi^{1}} \\
& -\left[\sqrt{g} a_{l}^{(2)} u^{l}\right]_{i j-1 / 2} \delta \xi^{1}=0 .
\end{aligned}
$$

In practice the contravariant base vectors $a_{l}^{(k)}$ are replaced by expressions in terms of the covariant basevectors, see Sec. 4.1. Some details regarding the discrete approximations used for the partial differentials in Eq. (23) and the covariant vectors are given in Appendix B.

By means of Taylor expansion of the discrete variables it can be shown that the resulting system of finite difference equations is consistent and forms a second-order approximation to the continuous (transformed) Stokes equations, i.e., the truncation error is $O\left((\delta \xi)^{2}\right)$ if $\delta \xi^{1}=\delta \xi^{2}=\delta \xi$.

4.3 Multigrid. If $N$ is the number of grid cells in each direction the linear system of equations resulting from the discretization of Eqs. (19), (20), and (21) for the case of a pressure boundary consists of $3 N^{2}$ equations and unknowns. To solve the system either a direct or an iterative method can be used. Both methods have the disadvantage of being computationally expensive and thereby limiting the number of cells for which the problem can be solved in acceptable CPU times. However, for the case of iterative methods this problem can be overcome if Multigrid (MG) techniques are used. The principle of MG techniques is to design an iterative scheme that efficiently reduces high frequency components of the error and use this to smooth the error. As this is a property of many iterative schemes this is usually not too difficult. With such a scheme after a few relaxations on the (target) grid the remaining smooth error can then be solved on a coarser grid on which it is less smooth and thus can be solved more efficiently. The approximation to the smooth error on this coarser grid can then be used to correct the approximation on the target grid. Applying this approach recursively leads to an algorithm where to solve the problem on a given target grid a set of coarser grids is used. The result is an iterative cycle which traverses a sequence of grids in a prescribed manner referred to as a coarse grid correction cycle. When properly designed such a cycle converges at a grid independent speed with the efficiency with which the high frequency components of the error are reduced by the iterative process. Typically an error reduction of an order of magnitude per cycle can be obtained, independent of the mesh size on the target grid. Multigrid techniques were originally introduced for scalar elliptic partial differential equations in the 1970s. Since then efficient Multigrid algorithms have been developed for a wide variety of problems in many fields in science. For more details the reader is referred to the many books, e.g., Brandt [49], Stüben and Trottenberg [50], Hackbusch [51], Briggs [52], Joppich [54], Wesseling [53] and for the specific application to lubrication problems to Venner and Lubrecht [55].

For many scalair partial differential boundary value problems the standard iterative processes such as Gauss-Seidel relaxation have good error smoothing properties. For systems of equations to find a suitable scheme is more involved due to the coupling between equations. For these problems one distinguishes uncoupled (SIMPLE, DGS) and coupled methods (see Wesseling [53] for good description of methods). For the Stokes equations considered here a symmetric coupled Gauss Seidel relaxation was used which is a robust and efficient smoother. In its simplest form the method consists of scanning the grid cell by cell at each cell solving the five unknowns in that cell simultaneously. This gives good smoothing when the problem is isotropic $(\epsilon=O(1))$. However, with decreasing $\epsilon$ the anisotropy increases and the equations become more strongly coupled in $z$ direction than in $x$ direction. As a result smoothing behavior in the $x$ direction will deteriorate. The remedy is to apply the relaxation in a "line relaxation" manner that is, instead of scanning the grid cell by cell to scan the grid in a linewise manner each time solving all equations of the cells of one vertical line simultaneously. As the system of equations for one line has a narrow band this can be done efficiently with a direct solver. The resulting line relaxation scheme still requires an amount of work proportional to the number of unknowns. For a detailed description of the system of equations to be solved per line the reader is referred to [42]. With this relaxation scheme in a Multigrid solver an excellent grid independent convergence rate for large and small $\epsilon$ has been achieved, see [42].

Finally, the interaction between the different grids in the Multigrid algorithm involves "inter-grid" transfers. In this work the inter-grid operators as described by Vanka [56] were used.

\section{Results}

Below results are presented obtained with the Stokes equations, the Reynolds equation and the first and second order perturbation equations for the model problem in two configurations.

5.1 Single Local Feature. For the case of a single local feature (SLF) in a uniform channel Eq. (13) and (14) were used with $h_{\mathrm{loc}}=1.0, n_{s}=0, \Lambda_{s}=\lambda_{s}, \kappa_{s}=16 / \lambda_{s}$ which gives a single valley asperity combination. The parameters varied are the wave- 


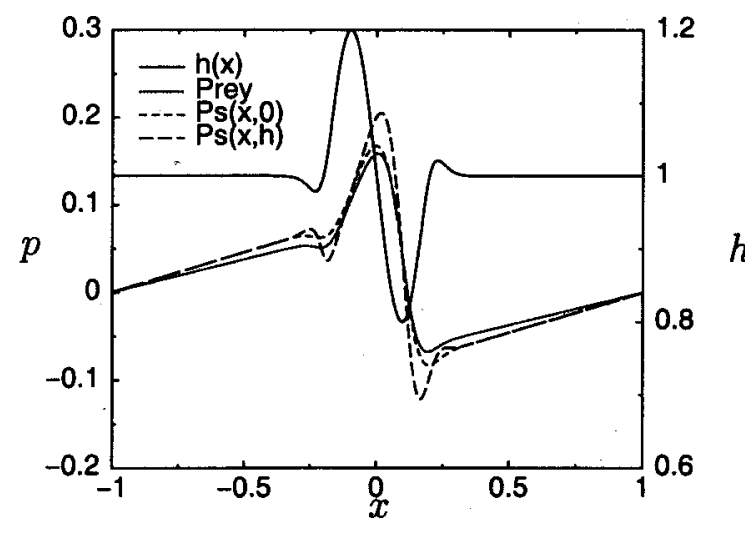

(a)

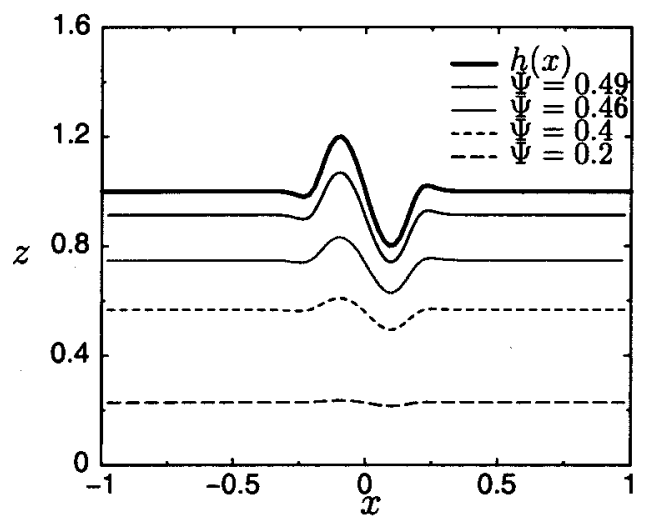

(b)

Fig. 3 (a) Pressure as function of $x$ for $z=0$ and $z=h(x)$; and (b) streamlines: both as function of $x$ and $z$ for $\epsilon=0.1, \lambda_{s}$ $=0.4, a_{s}=0.2$.

length $\lambda_{s}$, the amplitude $a_{s}$, and $\epsilon=H / L$, which appears in the Stokes equations. The reader is reminded that $a_{s}$ and $\lambda_{s}$ are dimensionless quantities. In physical terms the amplitude of the feature is $A=H a_{s}$ and its wavelength $\Lambda=L \lambda_{s}$.

In Fig. 3 the solutions to the problem are shown obtained for a typical case with the different modeling equations. In Fig. 3(a) the pressure at the lower and upper surface as a function of $x$ are shown according to the Stokes solution, and the pressure solution according to the Reynolds equation. In Fig. 3(b) the streamlines of the Stokes solution are shown. In Fig. 3(a) it can be seen that in the local region of the feature the Stokes solution clearly predicts a $z$-dependent pressure. However, the Reynolds and Stokes pressure solution do not only differ in the vicinity of the feature. Also in the uniform channel region before and after the local feature the Reynolds and Stokes pressure solution differ. So, in the Stokes solution the presence of the local feature is "felt" throughout the entire domain with its influence on the solution decreasing with increasing distance from the feature.

The streamlines in Fig. 3 indicate that the flow follows the boundaries. Near the surface $z=0$ the streamlines are straight, and near the surface $z=h(x)$ they align with the feature. In between there is a gradual transition. This is not always the case. The flow may exhibit recirculation. For this problem two types of recirculation can occur. The first type occurs for an amplitude $a_{s}>0.5$ and is characterized by a large scale back-flow. The feature in fact blocks the flow and eventually for very large amplitudes splits the problem into two separate problems. This global type of recirculation is also predicted by the Reynolds solution. It is illustrated in Fig. 4(a) where the Stokes velocity solution is shown for $\epsilon$ $=0.01, a_{s}=0.9$, and $\lambda_{s}=0.06$. As can be seen from Fig. 4 this

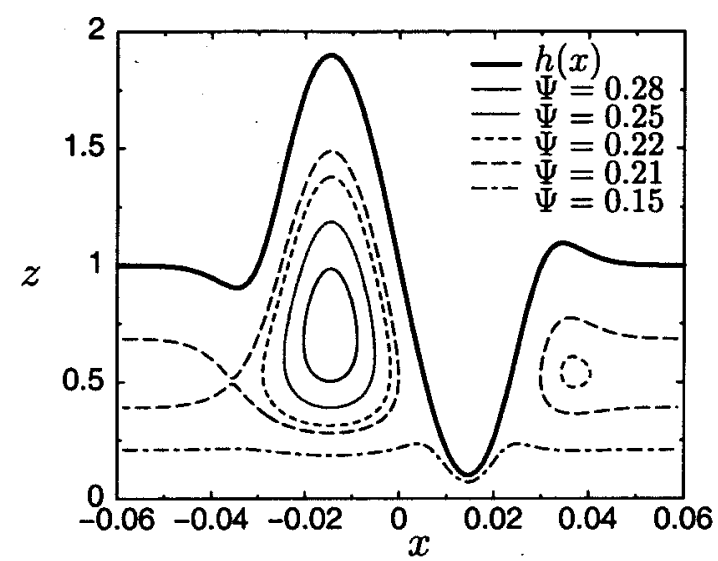

(a)

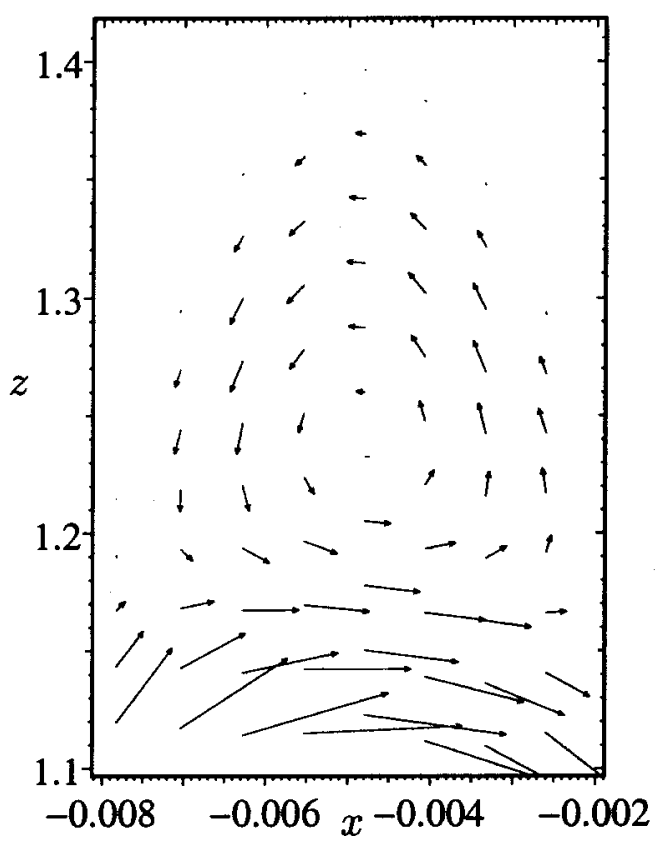

(b)

Fig. 4 Two types of recirculation for $\epsilon=0.01$ : (a) large amplitude induced recirculation $a_{s}=0.9$ and $\lambda_{s}=0.06$; and (b) short wavelength induced recirculation $a_{s}=0.4$ and $\lambda_{s}=0.02$.

amplitude induced recirculation has large effect on the flow. The recirculation zone extends all the way to the inflow boundary. The second type of recirculation is very local. It occurs in the valley of the feature when $\epsilon / \lambda_{s}=O(1)$ and is not predicted by the Reynolds equation. An illustration is given in Fig. 4(b). Because of its small size and the small value of the velocities it is only visible in a picture zooming in on the valley of the feature. This recirculation is of the same type as found in the case of a driven cavity problem as for example presented by Moffat [57]. The bulk of the fluid simply passes below the feature and the recirculation has a very small effect on the overall flow pattern. However, it can still be of importance as it implies that a certain amount of lubricant stays at the same position and is not replaced by new lubricant. Finally, a combination of both recirculation patterns can occur when $\epsilon / \lambda_{s}=O(1)$ and $a_{s}>0.5$.

To quantify the difference between the Reynolds and the Stokes solutions the difference of the generated load force of the solutions on the lower surface is taken, as defined by the quantity $\bar{R}$, 


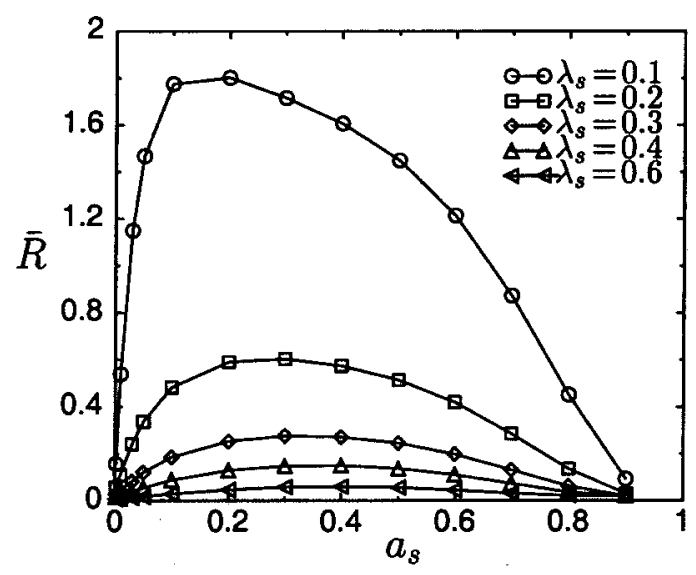

(a)

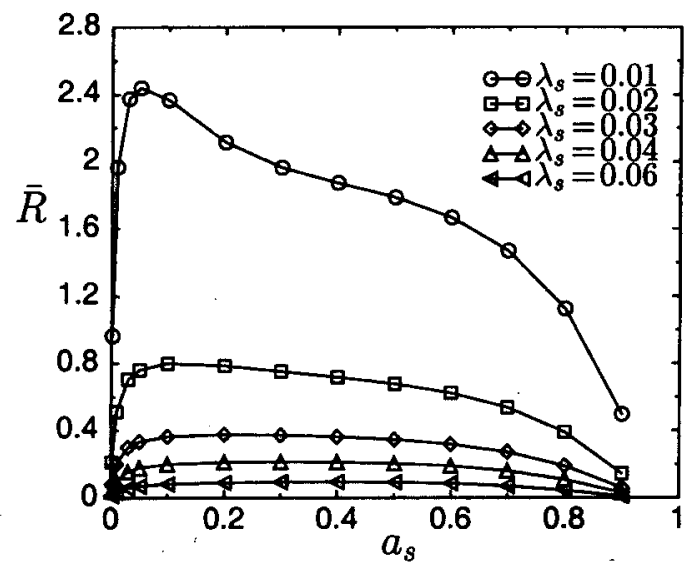

(b)

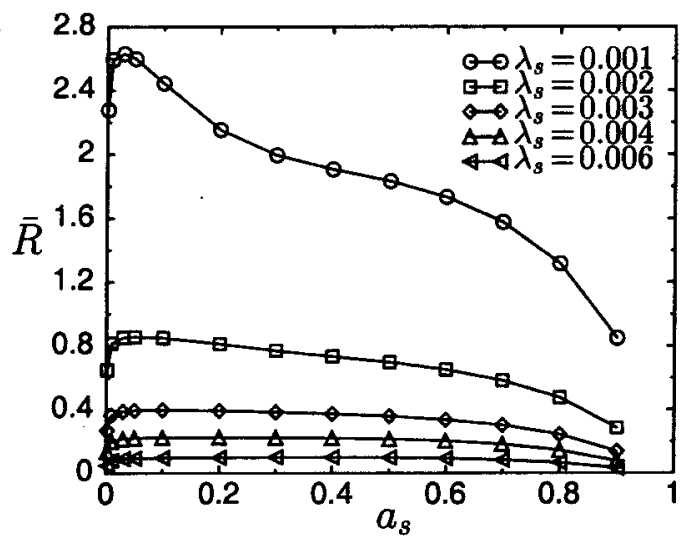

(c)

Fig. $5 \bar{R}$ as function of $a_{s}$ for (a) $\epsilon=0.1$; (b) $\epsilon=0.01$; and (c) $\epsilon=0.001$

see Eq. (17). For different values of $\epsilon(\epsilon=0.1,0.01,0.001)$ Fig. 5 shows the value of $\bar{R}$ as a function of $a_{s}$ and $\lambda_{s}$. This figure shows that $\bar{R}$ does not monotonously increase or decrease with $a_{s}$ but that it has a maximum. Only for sufficiently large $a_{s}$ it decreases with $a_{s}$. Also, for a smaller value of $\epsilon$ the maximum value of $\bar{R}$ occurs at a smaller value of $a_{s}$. Finally, for a given $a_{s}$ and $\epsilon$ the value of $\bar{R}$ decreases with increasing $\lambda_{s}$ as is expected.

Note that in each figure results are shown for values of $\lambda_{s}$ that differ, e.g., $0.1<\lambda_{s}<0.6$ for $\epsilon=0.1,0.01<\lambda_{s}<0.06$ for $\epsilon=0.01$ and $0.001<\lambda_{s}<0.006$ for $\epsilon=0.001$. This choice has been made to show that the magnitude of $\bar{R}$ depends mainly on the ratio $\epsilon / \lambda_{s}$. So, disregarding the detailed shape of each curve, its level is de-

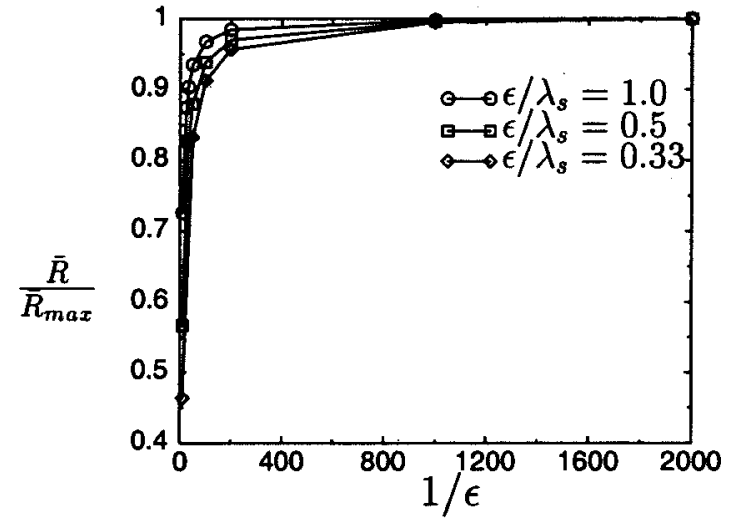

Fig. 6 Limit of $\bar{R} / \bar{R}_{\max }$ if $\epsilon / \lambda_{s}=$ constant and $\epsilon \rightarrow 0$, for $a_{s}=0.1$

termined by this ratio. The purpose of this study is to find a general rule indicating when a Reynolds solution is valid. For this problem one thus finds that the difference between the Stokes and the Reynolds solution is a function of $\epsilon / \lambda_{s}$. As $\epsilon=H / L$ and $\lambda_{s}$ $=\Lambda / L$ this means that in physical terms the accuracy of the Reynolds solution depends on the value of the ratio $H / \Lambda=$ film thickness/wavelength feature.

From the results presented here it is estimated that a difference of 10 percent between the Reynolds and the Stokes solution measured by $\bar{R}$ occurs for $\epsilon / \lambda_{s}=H / \Lambda>0.2$. The fact that the ratio $H / \Lambda$ determines the behavior is in line with expectations expressed in the literature, e.g., by Elrod [9]. More important is that now a quantification can be given. Significant differences between the load capacity according to the Reynolds solution and the load capacity according to the Stokes solution occur for $H / \Lambda$ $=O(0.1)$ and larger.

In Fig. 5 it can be seen that there appears to be a maximum value for the difference between the generated lift forces when $\epsilon / \lambda_{s}$ is kept constant and at the same time $\epsilon \rightarrow 0$. This behavior is visualised in Fig. 6. The figure shows $\bar{R}$ as a function of $\epsilon$ for three values of $\epsilon / \lambda_{s}$ fixed. To show the limiting behavior the results have been scaled on the maximum value of $\bar{R}$ that is observed which is the value reached at $\epsilon=0.005$. The observed behavior can be understood when translating back to the un-scaled variables. If $H$ is assumed to be constant, $\Lambda$ is constant: $\epsilon / \lambda_{s}$ $=H / L \lambda_{s}=H / \Lambda=$ constant. So, if $L \rightarrow \infty$ the distance between local feature and in/out flow boundary increases. The limiting behavior thus indicates, as anticipated, that there is no interaction between the surface feature and the in/out flow boundaries.

5.2 Perturbation SLF. As mentioned a perturbation approach can be used to obtain a correction to the Reynolds solution without having to solve the full Stokes equations. For the problem of a SLF such perturbation solutions are compared with the solution of the Reynolds equation and the solution of the Stokes equations. For a characteristic case, the accuracy and convergence of the perturbation in relation with the wavelength of the feature is studied.

Figure 7 shows some results obtained for the case $\epsilon=0.01, a_{s}$ $=0.4$ and two wavelengths $0.04,0.02$. The figures show the pressure at the surface $z=0$ and $z=h(x)$ as obtained from the Reynolds equation, the Stokes solution, and from two perturbation solutions: the first order and the second order. The global features of the SLF solution have already been discussed in detail in the previous section. Here only the solution in the vicinity of the feature is shown.

Consider the case $\lambda_{s}=0.04$. The first order perturbation solution to the pressure already differs very little from the Stokes solution, and the second order perturbation result is an even more accurate approximation to the Stokes solution. With decreasing wavelength the accuracy of the perturbed solutions is expected to 

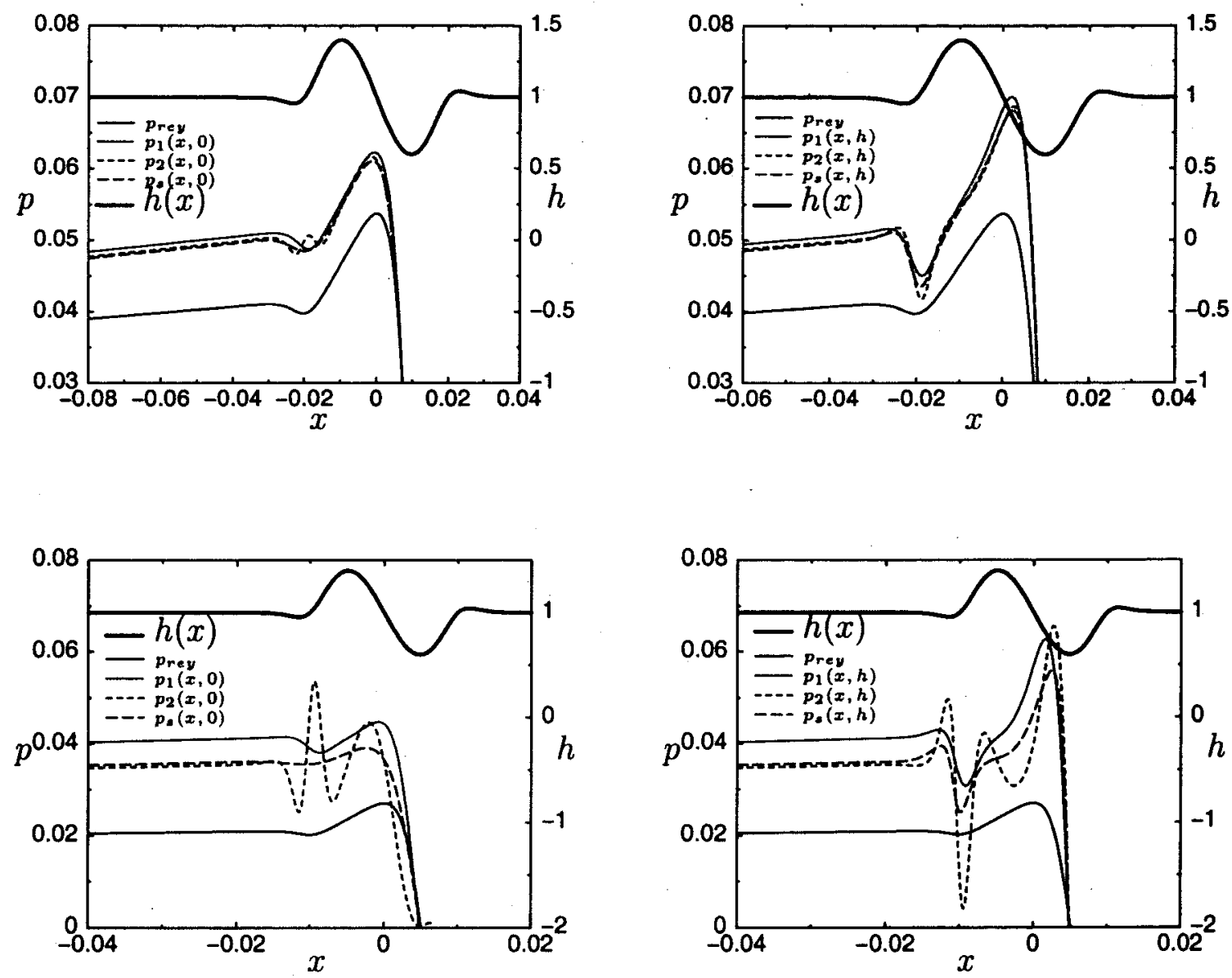

Fig. 7 Pressure at the surface $z=0$ (left) and $z=h(x)$ (right) for the Reynolds solution ( $p_{\text {rey }}$ ), the Stokes solution $\left(p_{s}\right)$ and a first $\left(p_{1}\right)$ and second order $\left(p_{2}\right)$ perturbation solution for the case $\epsilon=0.01$ and $a_{s}=0.4$ while $\lambda_{s}$ $=0.04$ (top) and $\lambda_{s}=0.02$ (bottom)

decrease. This can be seen from the results obtained for smaller wavelengths. For $\lambda_{s}=0.02$ the global correction provided by the perturbation is accurate in the region away from the feature but locally near the feature it is very poor. The perturbation solutions contain an erroneous local oscillation with an amplitude that for the second order result is even larger than in the first order result.

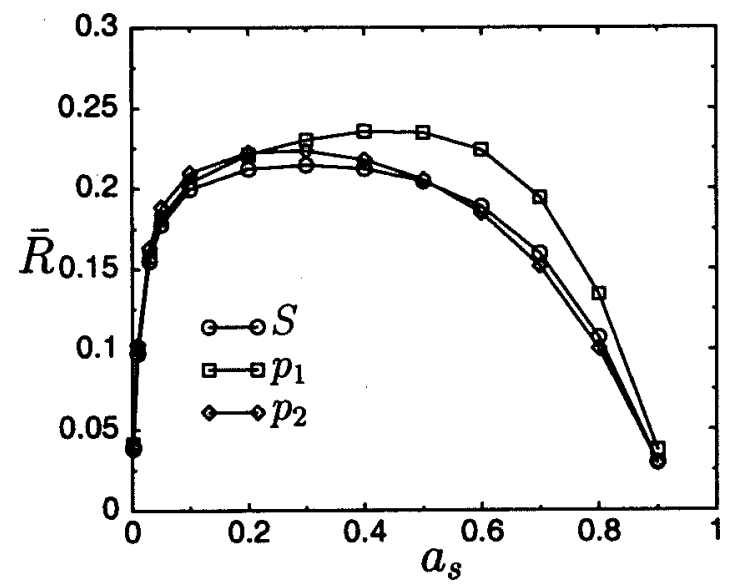

Fig. 8 The value of $\overline{\boldsymbol{R}}$ for the Stokes (S) solution, the first order perturbation $\left(p_{1}\right)$ solution and the second order perturbation $\left(p_{2}\right)$ solution for the SLF with $\epsilon=0.01$ and $\lambda_{s}=0.04$
This indicates that locally the perturbation in $\epsilon$ does not converge. The local gradients have become too large for the global parameter $\epsilon$ to be an adequate perturbation parameter.

It is observed that both cases $\left(\lambda_{s}=0.04,0.02\right)$ the Stokes pressure is larger than the Reynolds pressure. A closer look at the pressure expansion, Eq. (12) shows that this is caused by $\widetilde{p}_{1}(x)$ and $\widetilde{p}_{2}(x)$ because $w_{0}$ and $w_{1}$ only differ from zero in the region where $d h / d x$ is nonzero. The equations for $\widetilde{p}_{1}(x)$ and $\widetilde{p}_{2}(x)$ contain derivatives of $h$ with respect to $x$, this is in contrast to the Reynolds equation. So, the curvature of the feature does not only have a local effect but also a global effect.

The behavior described above is also reflected in global parameters of the solution such as the $\bar{R}$ value used to measure the difference between the Stokes solution and the Reynolds solution. This is illustrated in Fig. 8. In this figure $\bar{R}$ is shown as a function of the amplitude $a_{s}$ for the case of $\epsilon=0.01, \lambda_{s}=0.04$ for three solutions. First the value obtained comparing the Stokes and the Reynolds solution. Secondly the value obtained comparing the first order perturbation solution to the Reynolds solution and finally the value obtained comparing the second order perturbation solution to the Reynolds solution. From the figure it is concluded that for this case, even when the amplitude is large, the value of $\bar{R}$ obtained using the perturbation solutions is an accurate approximation to the value obtained using the complete Stokes solution.

With respect to the calculated velocity profiles it is noted that as was mentioned in Section 4.1 a recirculation induced by a small wavelength can not be predicted by the series expansion in terms of $\epsilon$. 


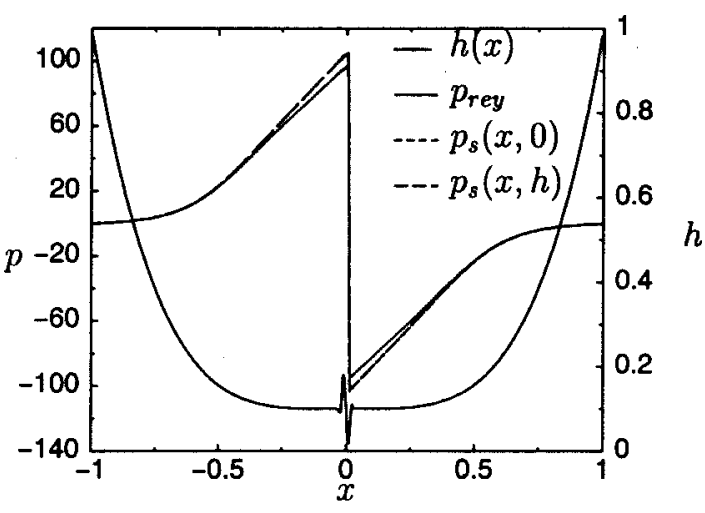

(a)

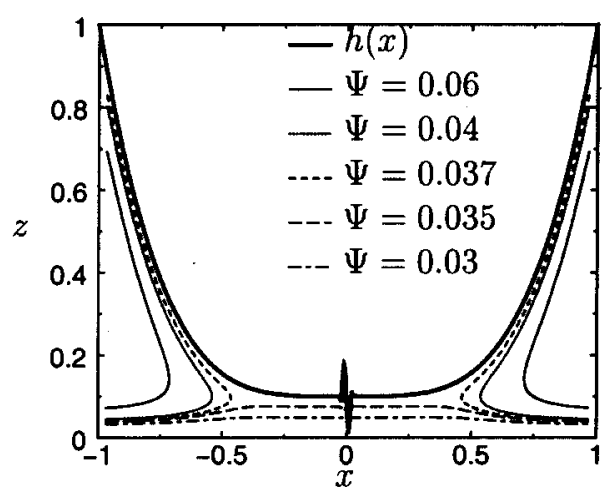

(b)

Fig. 9 (a) Pressure as function of $x$ for $z=0$ and $z=h(x)$; and (b) streamlines: both as function of $x$ and $z$ for $\epsilon=0.1, h_{\text {loc }}$ $=0.1, \lambda_{s}=0.04, a_{s}=0.08$.

In conclusion, it has been shown that for the SLF problem, the perturbation theory yields accurate corrections to the Reynolds solution as long as the ratio $\epsilon$ to wavelength is not too large. For large values of $\epsilon / \lambda_{s}$ the global correction to the pressure solution will still be reasonable, but the predicted local pressure and velocity field around the feature are inaccurate. In such cases better results may be obtained with a more sophisticated perturbation approach, see Sec. 2.

5.3 EHL Relevant Geometry. Unlike the SLF problem discussed so far, the surfaces in a lubricated contact are not nominally flat. In this sense the SLF problem is not realistic for a full scale contact. However, it can still be representative for the local situation inside a contact, but then the boundary pressure for the local problem should be taken from the global pressure of the large scale solution.

A larger scale problem combined with a local feature is considered in this section. In tribology often the contacting surfaces are approximated by a polynomial of the second degree. In this section a contact geometry is modeled by a $4^{\text {th }}$ degree polynomial to create a situation that resembles the usual parabola but with a flat region in the center. The resulting film geometry can be seen as an approximation of the film in EHL contacts where the flat region is the result of the deformation.

To describe the upper boundary, Eqs. (13) and (14) were used with $n_{s}=4, h_{\text {loc }} \Lambda_{s}=\lambda_{s}$ and $\kappa_{s}=16 / \lambda_{s}$. The remaining parameters to be varied are $h_{\text {loc }}$ the local film thickness in the flat region, $\epsilon, a_{s}$, and $\lambda_{s}$. The boundary conditions are the same as for the SLF.

In Fig. 9 the different (Reynolds, Stokes, perturbation) solutions are shown for a typical case. In Fig. $9(a)$ are shown the computed

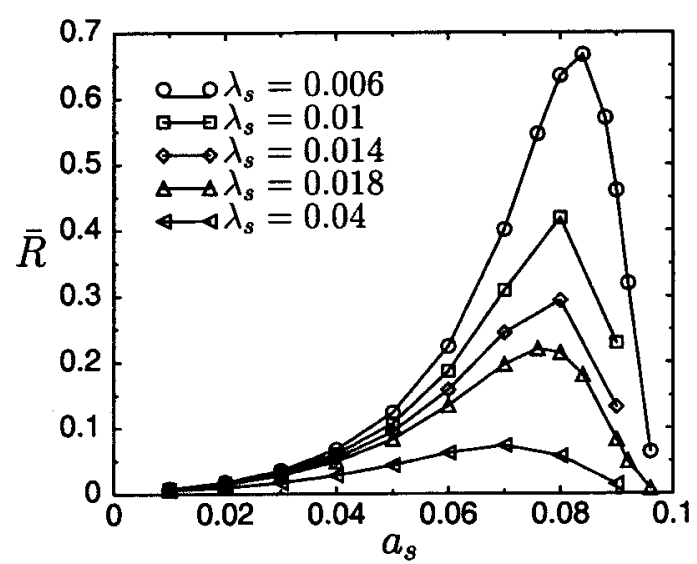

(a)

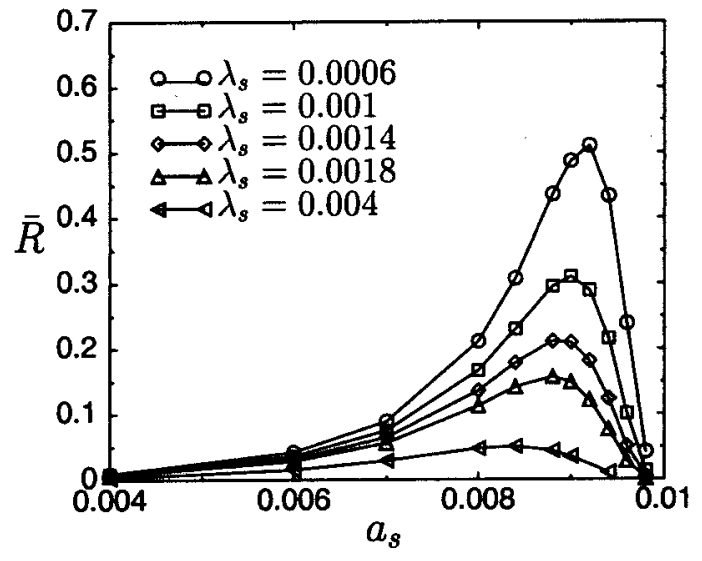

(b)

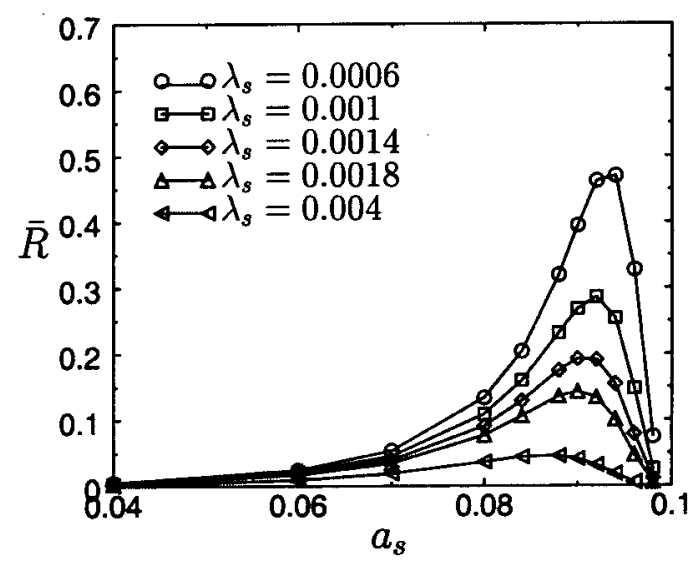

(c)

Fig. 10 The value of $\bar{R}$ as function of $a_{s}$ for different $\lambda_{s}:$ (a) $\epsilon=0.1$ and $h_{\mathrm{loc}}=0.1 ;$ (b) $\epsilon=0.1$ and $h_{\mathrm{loc}}=0.01$; and $(c) \epsilon=0.01$ and $h_{\text {loc }}=0.1$.

pressure at the surfaces $z=0$ and $z=h$ as a function of $x$ for the Stokes solution and the pressure according to the Reynolds equation. Figure $9(b)$ shows the flow pattern in the gap. In Fig. $9(a)$ it can be seen that the Stokes pressure is larger than the Reynolds pressure as was found for the SLF. However, the Stokes pressures at the lower and upper surface hardly differ indicating very weak $z$ dependence, except for the region near the local feature. The stream lines in Fig. 9(b) show that at the entrance and at the exit of the contact recirculation occurs. In addition there is local recirculation in the valley of the feature, although this is not visible in Fig. $9(b)$. 

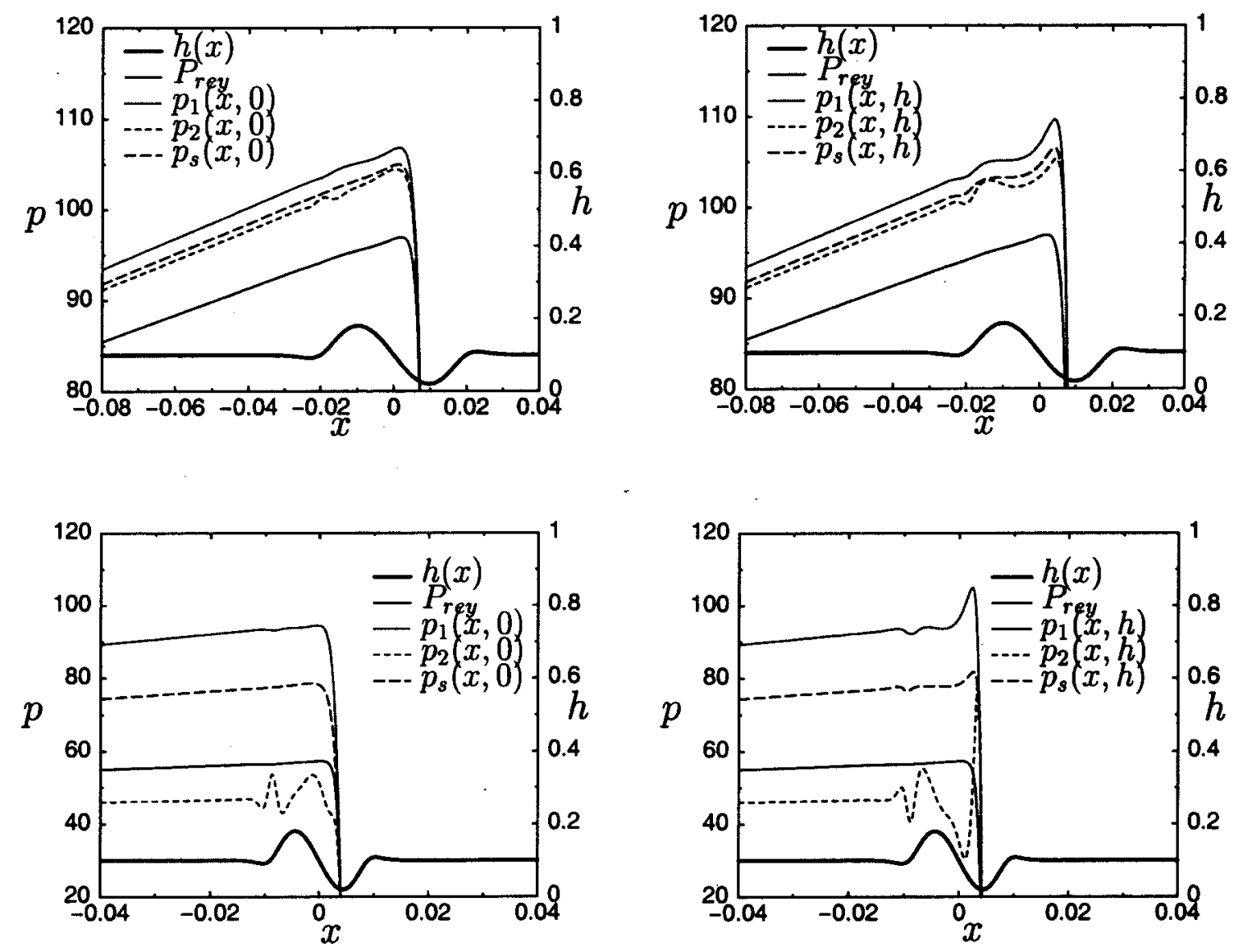

Fig. 11 Pressure at the surface $z=0$ (left) and $z=h(x)$ (right) for the Reynolds solution ( $p_{\text {rey }}$ ), the Stokes solution $\left(p_{s}\right)$ and a first- $\left(p_{1}\right)$ and second order $\left(p_{2}\right)$ perturbation solution for the case $\lambda_{s}=0.04$ (top) and $\lambda_{s}$ $=0.018$ (bottom) while for both cases $\epsilon=0.1, h_{\mathrm{loc}}=0.1$, and $a_{s}=0.08$

As was done for the SLF, the difference in load capacity $\bar{R}$ has been calculated to quantify the difference between the Stokes and the Reynolds solution. Results for three combinations of $\epsilon$ and $h_{\text {loc }}$ are shown in Fig. $10(a-c) . \epsilon=0.1$ and $h_{\text {loc }}=0.1, \epsilon=0.01$ and $h_{\mathrm{loc}}=0.1$, and $\epsilon=0.1$ with $h_{\mathrm{loc}}=0.01$. The dependence of the value of $\bar{R}$ on the feature amplitude $a_{s}$ is shown for different values of the feature wavelength $\lambda_{s}$. It can be seen that with decreasing $\lambda_{s}$ the accuracy of the Reynolds solution decreases, as was found for the SLF. However, in contrast to the SLF results, here only for large amplitudes a significant difference between the Stokes and Reynolds solution occurs. For the cases shown in Fig. 10 the wavelengths are chosen such that the factor $\epsilon h_{\mathrm{loc}} / \lambda_{s}$ is the same. It is clear that the graphs in the figures Figure $10(a-c)$ are very similar and the predicted values of $\bar{R}$ differ little. The main difference is between the first case, $\left(\epsilon=0.1\right.$ and $\left.h_{\mathrm{loc}}=0.01\right)$ and the other two. This suggests that for the larger $\epsilon$ the results are also effected by interaction between the in- and out-flow boundaries and the feature.

In conclusion, the behavior of the difference between the Reynolds solution and the Stokes solution for this problem appears to be determined by the ratio $\epsilon h_{1 \mathrm{oc}} / \lambda_{s}$. The influence of this ratio can be understood as follows. From the perturbation analyses it follows that $d h / d x$ and higher derivatives are important and can completely undo the effect of a small $\epsilon$ in a series expansion. However, the value $\epsilon d h /\left.d x\right|_{x=0} \approx-2 \pi a_{s} \epsilon / \lambda_{s} \sim a_{s} \epsilon / \lambda_{s}$ is not the right parameter to indicate if the solutions of the Reynolds equation are accurate. This already follows from the parabolic shape of the curves in Fig. 10: In each graph the same value of $\bar{R}$ is obtained for two different values of $a_{s} \epsilon / \lambda_{s}$. But, taking the maximum value one obtains:

$$
\left.\epsilon \frac{d h}{d x}\right|_{\max } \approx-\frac{2 \pi \epsilon h_{\mathrm{loc}}}{\lambda_{s}} \sim \frac{\epsilon h_{\mathrm{loc}}}{\lambda_{s}}
$$

when $a_{s} \rightarrow h_{\mathrm{loc}}$. In physical terms $\epsilon h_{\mathrm{loc}} / \lambda_{s}=H h_{\mathrm{loc}} / L \lambda_{s}$ $=H_{\mathrm{loc}} / \Lambda$ so it is the local ratio of film thickness to wavelength that determines the effect of the feature which confirms the hypothesis of Elrod [9] and is consistent with the findings for the SLF problem. Measured by means of the load capacity a difference in excess of 10 percent between the Stokes and the Reynolds solution was found to occur for $H_{10 c} / \Lambda>0.25$ and the maximum difference was found for an amplitude close to the local film thickness.

5.4 Perturbation EHL Relevant Geometry. In Fig. 11 some results are shown for the case $\epsilon=0.1, h_{\mathrm{loc}}=0.1, a_{s}=0.08$ and two wavelengths $\lambda_{s}=0.04,0.018$. The figures show the pressure at the surface $z=0$ and $z=h(x)$ as obtained from the Reynolds equation, the Stokes solution, and the first and second order perturbation solution. As the global features of the solution have been discussed in detail in the previous section, here only the solution in the region around the feature is shown. Consider the case $\lambda_{s}=0.04$. The first order perturbation solution is already an excellent improvement on the Reynolds solution. The secondorder perturbation result is even more accurate. Clearly the perturbation in $\epsilon$ for this case converges. With decreasing wavelength the accuracy of the perturbed solutions is expected to decrease. This can be seen from the results shown for a smaller wavelength. For $\lambda_{s}=0.018$ the perturbation solution is incorrect not only in the region of the feature but also on a global scale. Also with increasing perturbation order the series expansion diverges further from 


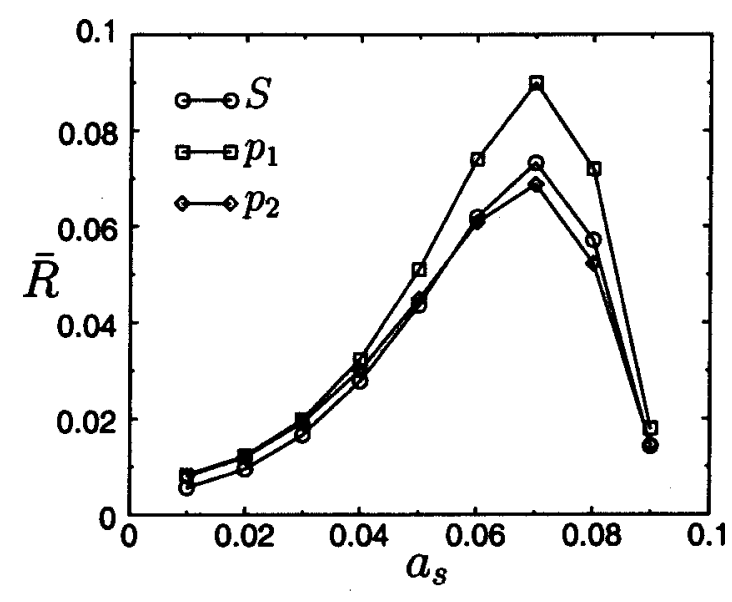

Fig. 12 The value of $\overline{\boldsymbol{R}}$ for the EHL geometry with local feature obtained for the case of the Stokes $(S)$ solution the first order perturbation solution $\left(p_{1}\right)$ and the second order perturbation solution $\left(p_{2}\right) . \epsilon=0.1, h_{\mathrm{loc}}=0.1$ and $\lambda_{s}=0.04$.

the Stokes solution indicating that for this case a perturbation based only on the single global parameter $\epsilon$ is no longer the adequate choice, see Sec. 2. It is observed that in both cases $\left(\lambda_{s}\right.$ $=0.04,0.018)$ the Stokes pressure exceeds the Reynolds pressure as was found for the SLF.

When the perturbation converges also the difference between the generated load predicted by the Reynolds solution and the Stokes solution is predicted quite accurately by the perturbation solutions. In Fig. 12 for the case $\epsilon=0.1, h_{\text {loc }}=0.1$ and $\lambda_{s}=0.04$ the value of $\bar{R}$ is shown as a function of the amplitude for three solutions: the first-order and second-order perturbation solutions and for the Stokes solution. It can be seen that a higher-order series expansion gives a better prediction. From the figure it can be concluded that a difference of about 10 percent in predicted load capacity can be corrected with the perturbation approach. However, this is less than for the SLF where a deviation up to 20 percent between the Stokes solution and the Reynolds equation could be corrected accurately with the perturbation analysis.

\section{Conclusion}

A numerical algorithm was developed for the solution of the flow in a thin film based on the Stokes equations. The equations were transformed using curvi-linear coordinates and discretized using a staggered grid arrangement. The discrete system of equations is solved with symmetric coupled Gauss Seidel relaxation and Multigrid techniques are used to accelerate convergence. To obtain typical multigrid efficiency of grid independent convergence it is essential that the relaxation be applied in a line relaxation manner. This is due to the anisotropical nature of the problem of flow in a thin film.

The algorithm was applied to the solution of the model problem of the flow in a narrow gap with a single local surface feature for two cases with different global geometry of the gap. The computed velocity and pressure fields were compared with the solutions obtained when the problem is modelled with the Reynolds equation. It is shown that the magnitude of the differences between the Reynolds and Stokes solutions depends on the local ratio film thickness to feature wavelength. Large differences in an integrated quantity such as the predicted load occur when this ratio is $O(0.1)$. However, at a local scale in one of the field quantities velocity or pressure large differences may already occur at smaller values. This may be important when effects on surface deformation or surface fatigue are to be estimated.

Because solutions to the Stokes equations are still not available for many practical lubrication problems, a simple tool to obtain an estimate of the error in a Reynolds model solution using only
Reynolds type of equations for which many solvers exist can be of great convenience. This can be achieved with a perturbation approach. For the model problems considered here it is shown that a first and second order correction can be computed which are quite accurate but this depends on the perturbation parameter that is used. A straightforward perturbation in terms of the global film aspect ratio will break down when the slopes in the film locally are too large.

In this paper the validity of the solution was investigated in relation to the geometry of the (local) gap. Another limitation to the validity of the Reynolds solutions is formed by the piezoviscous behavior [41], and the compressibility behavior of the lubricant. These effects also lead to a cross-film dependence of the pressure that can not be predicted with the Reynolds equation and are described in [42].

\section{A Perturbation}

In this appendix the expansion of the Stokes equation in $\gamma$ ( $=\epsilon^{2}$ ) will be carried out up to second order for the configuration assumed in the model problems, i.e., a lower surface $z=0$ moving with speed $u_{c}$ and a stationary upper surface $z=h$. The related velocity boundary conditions are assumed to be satisfied by the zero order solution: $u_{0}(x, 0)=1, w_{0}(x, 0)=0$ and $u_{0}(x, h)=0$, $w_{0}(x, h)=0$.

Before presenting the equations it is noted that for the present case of iso-viscous, incompressible flow, the perturbation solutions can be obtained relatively easily. However, for the more general case of a pressure-dependent density and viscosity the entire series expansion is much more complicated.

The equations for the first order terms are the same as Eqs. (6), (7) and (8), but with a the right-hand side now formed by derivatives of the zero $^{\text {th }}$-order solution.

$$
\begin{gathered}
\frac{\partial p_{1}}{\partial x}-\frac{\partial^{2} u_{1}}{\partial z^{2}}=\frac{\partial^{2} u_{0}}{\partial x^{2}}, \\
\frac{\partial p_{1}}{\partial z}=\frac{\partial^{2} w_{0}}{\partial z^{2}}, \\
\frac{\partial u_{1}}{\partial x}+\frac{\partial w_{1}}{\partial z}=0 .
\end{gathered}
$$

The Eqs. (26), (27) and Eq. (28) can be solved in the same way as by Eqs. (6), (7) and (8). Integration of Eq. (27) with respect to $z$ gives an expression for the pressure correction,

$$
p_{1}=\frac{\partial w_{0}}{\partial z}+\widetilde{r}_{1}(x)
$$

Integrating Eq. (26) twice with respect to $z$ results in an expression for $u_{1}$. The unknown variable $p_{1}$ in the expression for $u_{1}$ follows from Eq. (29). After integration of Eq. (28) with respect to $z$ and substitution of the expression for $u_{1}$ and the correct boundary values, an equation for $\tilde{p}_{1}$ follows:

$$
\begin{gathered}
\frac{d}{d x}\left[\left(\frac{d h}{d x}\right)^{2}\left(6 c_{1}+28 h(x)\right)+\frac{d^{2} h}{d x^{2}} h(x)\left(c_{1}+4 h(x)\right)\right. \\
\left.-10 h(x)^{3} \frac{d \widetilde{p}_{1}}{d x}\right]=0 .
\end{gathered}
$$

where $c_{1}$ is an integration constant ${ }^{1}$ resulting from integration of Eq. (10). As $p_{0}=p_{0}(x)$ it is clear that the $z$-dependency in the first order correction comes from $\partial w_{0} / \partial z$ which is already known when the Reynolds solution is available.

\footnotetext{
${ }^{1} d p_{0} / d x=6 h(x)+c_{1} / h^{3}(x)$
} 
The second order corrections follow from substituting Eq. (5) in Eqs. (2), (3) and (4) and equating second order terms in $\gamma^{2}$.

$$
\begin{aligned}
& \frac{\partial p_{2}}{\partial x}-\frac{\partial^{2} u_{2}}{\partial z^{2}}=\frac{\partial^{2} u_{1}}{\partial x^{2}}, \\
& \frac{\partial p_{2}}{\partial z}=\frac{\partial^{2} w_{1}}{\partial z^{2}}+\frac{\partial^{2} w_{0}}{\partial x^{2}},
\end{aligned}
$$

$$
\frac{\partial u_{2}}{\partial x}+\frac{\partial w_{2}}{\partial z}=0
$$

On the four boundaries of the domain the second order corrections should satisfy: $u_{2}=0, w_{2}=0, p_{2}=0$. Note that the righthand sides in the second order equations contain the lower order results which are all known. $w_{0}$ is given in Eq. (11). The first order perturbation $u_{1}$ follows from integrating Eq. (26) twice with respect to $z$ and is given by:

$$
u_{1}=\frac{c_{2} z(z-h)}{2 h^{3}}+\frac{z(z-h)\left(-30 c_{1} z^{2}-90 z^{2} h+9 c_{1} h^{2}-10 z h^{2}+32 h^{3}\right)\left(h^{\prime}\right)^{2}}{30 h^{5}}+\frac{z\left(c_{1}+4 h\right)\left(15 z^{3}-20 z^{2} h+3 z h^{2}+2 h^{3}\right) h^{\prime \prime}}{60 h^{4}},
$$

where $c_{1}$ and $c_{2}$ are integration constants following from the integration of Eq. (10) and Eq. (30) with respect to $x$ from -1 to 1 . For convenience the short-hand notations $h^{\prime}=d h / d x, h^{\prime \prime}=d^{2} h / d x^{2}$ etc. have been introduced. An expression for $w_{1}$ can be from Eq. (28) using the explicit form of $u_{1}$.

$$
\begin{aligned}
w_{1}= & \frac{z^{2}(z-h)\left(-30 c_{1} z^{2}-72 z^{2} h+9 c_{1} h^{2}-12 z h^{2}+16 h^{3}\right)\left(h^{\prime}\right)^{3}}{30 h^{6}}+\frac{c_{2} z^{2}(z-h) h^{\prime}}{2 h^{4}} \\
& +\frac{z^{2}(z-h)\left(36 c_{1} z^{2}-9 c_{1} z h+108 z^{2} h-19 c_{1} h^{2}-12 z h^{2}-64 h^{3}\right) h^{\prime} h^{\prime \prime}}{60 h^{5}}-\frac{z^{2}(z-h)^{2}(3 z+h)\left(c_{1}+4 h\right) h^{\prime \prime \prime}}{60 h^{4}} .
\end{aligned}
$$

The integration of Eq. (32) with respect to $z$ gives an expression for the pressure correction $p_{2}$ :

$$
p_{2}=\int\left[\frac{\partial^{2} w_{0}}{\partial x^{2}}+\frac{\partial^{2} w_{1}}{\partial z^{2}}\right] d z+\widetilde{p}_{2}(x)
$$

where $\widetilde{p}_{2}$ is unknown. An explicit expression for $p_{2}$ can now be found by substituting $w_{0}$ and $w_{1}$ in Eq. (36).

$$
\begin{aligned}
p_{2}= & \widetilde{p}_{2}(x)+\frac{z c_{2} h^{\prime}(3 z-2 h)}{2 h^{4}}+\frac{z\left(h^{\prime}\right)^{3}\left(-75 c_{1} z^{3}+60 c_{1} z^{2} h-180 z^{3} h+27 c_{1} z h^{2}+120 z^{2} h^{2}-18 c_{1} h^{3}+84 z h^{3}-32 h^{4}\right)}{30 h^{6}} \\
& +\frac{z h^{\prime} h^{\prime \prime}\left(45 c_{1} z^{3}-45 c_{1} z^{2} h+135 z^{3} h-15 c_{1} z h^{2}-120 z^{2} h^{2}+19 c_{1} h^{3}-78 z h^{3}+64 h^{4}\right)}{30 h^{5}} \\
& +\frac{z h^{\prime \prime \prime}\left(c_{1}+4 h\right)\left(-15 z^{3}+20 z^{2} h-6 z h^{2}-4 h^{3}\right)}{120 h^{4}} .
\end{aligned}
$$

Upon substitution of Eq. (37) in Eq. (31) and double integration with respect to $z$ an expression for $u_{2}$ can be found. This $u_{2}$ can be substituted in Eq. (33). This equation can be integrated with respect to $z$ between 0 and $h(x)$. Using the boundary conditions and the Leibnitz rule the following equation can be deduced:

$$
\begin{aligned}
\frac{d}{d x}[ & -h^{3} \frac{d \widetilde{p}_{2}}{d x}+\frac{-4\left(h^{\prime}\right)^{4}\left(9 c_{1}+46 h\right)}{525}+\frac{c_{2} h h^{\prime \prime}}{10} \\
& -\frac{h^{2}\left(h^{\prime \prime}\right)^{2}\left(80 c_{1}+107 h\right)}{1050}+\frac{3 c_{2}\left(h^{\prime}\right)^{2}}{5} \\
& -\frac{118 h\left(h^{\prime}\right)^{2} h^{\prime \prime}\left(c_{1}+8 h\right)}{525}-\frac{2 h^{2} h^{\prime} h^{\prime \prime \prime}\left(22 c_{1}+17 h\right)}{525} \\
& \left.+\frac{71 h^{3} h^{\prime \prime \prime \prime}\left(c_{1}+4 h\right)}{4200}\right]=0 .
\end{aligned}
$$

This is again a Reynolds type of equation for the unknown function $\widetilde{p}_{2}$. It can be solved by numerical integration.

Finally, the present case of iso-viscous, incompressible flow, the perturbation solutions can be obtained relatively easily. It should be noted that for the more general case of a pressure-dependent density and viscosity the entire series expansion is much more complicated.

\section{B Discretization}

The discretization of the partial derivatives in Eq. (23) is carried out by taking the average of the partial differential over a control volume $\Omega$. In the viscous part of Eq. (23) there are two types of terms. First, those where the variables are defined at the location where they are needed. For example:

$$
\begin{aligned}
\left\{\frac{\partial u}{\partial \xi^{1}}\right\}_{i j} & \equiv \frac{1}{\Omega} \int \frac{\partial u}{\partial \xi^{1}} d \Omega \approx \frac{1}{\Omega} \int \frac{\left(u_{i+1 / 2 j}-u_{i-1 / 2 j}\right)}{\delta \xi^{1}} d \xi^{1} d \xi^{2} \\
& =\frac{u_{i+1 / 2 j}-u_{i-1 / 2 j}}{\delta \xi^{1}},
\end{aligned}
$$

where it is assumed that within $\Omega u_{i+1 / 2 j}-u_{i-1 / 2 j}$ is constant along $\xi^{2}$. Second, the variables are not defined at the location where they are needed. For example:

$$
\begin{aligned}
\left\{\frac{\partial u}{\partial \xi^{2}}\right\}_{i j} & \equiv \frac{1}{\Omega} \int \frac{\partial u}{\partial \xi^{2}} \delta \Omega \\
& \approx \frac{1}{\Omega} \int \frac{\left(u_{i j+1 / 2}-u_{i j-1 / 2}\right)}{\delta \xi^{2}} d \xi^{1} d \xi^{2} \\
& =\frac{u_{i-1 / 2 j+1}+u_{i+1 / 2 j+1}-u_{i-1 / 2 j-1}-u_{i+1 / 2 j-1}}{4 \delta \xi^{2}} .
\end{aligned}
$$


We take $u_{i j+1 / 2}=1 / 4\left(u_{i-1 / 2 j}+u_{i-1 / 2 j+1}+u_{i+1 / 2 j+1}+u_{i+1 / 2 j}\right)$ and the shifted similar expression for $u_{i j-1 / 2}$. Regarding the pressure terms in Eq. (23), a similar approach is used, i.e., if the value of $p$ is needed in a point at which it is not defined, it is determined by interpolation from the surrounding values. This gives for linear interpolation:

$$
p_{i-1 / 2 j+1 / 2}=\frac{1}{4}\left(p_{i j}+p_{i-1 j}+p_{i j+1}+p_{i-1 j+1}\right) .
$$

In the discretization of the other equations we do not encounter any difficulties. For more details concerning the discretization see [42]. After discretization of the Stokes equations a linear system of equations arises.

The covariant base vectors, $\mathbf{a}_{(k)}$, can be calculated directly if the analytic expressions for $x, z$ as function of $\xi^{1}, \xi^{2}$ are known. But then the following identity will not hold in a transformed coordinate system:

$$
\int_{\Omega} \nabla \cdot \mathbf{u} d V=\oint_{\delta \Omega} \mathbf{u} \cdot d \mathbf{s}=0,
$$

where $\mathbf{u}$ is a constant vector field. Therefore a numerical approximation is used to calculate the base vectors. Because $x$ is only a function of $\xi^{1}$ and $z$ is scaled with the height $h\left(\xi^{1}\right)$, there are two types of approximations for each covariant base vector,

$$
\left\{\mathbf{a}_{(1)}^{1}\right\}_{i}=\frac{\left(x_{i+1 / 2}-x_{i-1 / 2}\right)}{\delta \xi^{1}}, \quad\left\{\mathbf{a}_{(1)}^{1}\right\}_{i-1 / 2}=\frac{\left(x_{i+1 / 2}-x_{i-3 / 2}\right)}{2 \delta \xi^{1}} .
$$

For $\mathbf{a}_{(1)}^{2}$ the approximation is found to be:

$$
\begin{aligned}
\left\{\mathbf{a}_{(1)}^{2}\right\}_{i, j} & =\xi_{j}^{2} \frac{\left(h_{i+1 / 2}-h_{i-1 / 2}\right)}{\delta \xi^{1}}, \quad\left\{\mathbf{a}_{(1)}^{2}\right\}_{i-1 / 2, j} \\
& =\xi_{j}^{2} \frac{\left(h_{i+1 / 2}-h_{i-3 / 2}\right)}{2 \delta \xi^{1}} .
\end{aligned}
$$

And for $\mathbf{a}_{(2)}^{2}$ the expression is:

$$
\left\{\mathbf{a}_{(2)}^{2}\right\}_{i}=\frac{1}{2}\left(h_{i-1 / 2}+h_{i+1 / 2}\right), \quad\left\{\mathbf{a}_{(2)}^{2}\right\}_{i-1 / 2}=h_{i-1 / 2} .
$$

The reader can verify that the contour integral in Eq. (42) is exactly zero with the above approximations for the covariant base vectors.

\section{Nomenclature}

(If the dimension is not stated the variable or parameter is dimensionless.)

$$
\begin{aligned}
A & =\text { amplitude of feature }[\mathrm{m}] \\
a_{s} & =\text { amplitude of sinusoidal feature } \\
a_{s}^{\prime} & =\text { amplitude term surface feature } \\
\mathbf{a}^{(k)} & =\text { contra-variant base vector } \mathbf{a}^{(k)}=\nabla \xi^{k} \\
\mathbf{a}_{(k)} & =\text { co-variant basis vector } \mathbf{a}_{(k)}=\partial x / \partial \xi^{k} \\
a_{l}^{(k)} & =l=1,2 \text { component of contra-variant base vector } \\
a_{(k)}^{l} & =l=1,2 \text { component of co-variant base vector } \\
e & =2.7182818 \\
\mathbf{f}^{h} & =\text { discrete righthand side vector } \\
\sqrt{g} & =\text { determinant Jacobian } \sqrt{g}=\operatorname{det}(J)=a_{(1)}^{1} a_{(2)}^{2}-a_{(2)}^{1} a_{(1)}^{2} \\
h(x) & =\text { surface geometry } \\
h_{\text {loc }} & =\text { local film thickness } \\
H & =\text { nominal film thickness }[\mathrm{m}] \\
H_{\text {loc }} & =\text { local film thickness }[\mathrm{m}] \\
i, j & =\text { grid index } \\
J & =\text { Jacobian of coordinate transformation } \\
L & =\text { film length }[\mathrm{m}] \\
n_{s} & =\text { film shape parameter } \\
\mathbf{n} & =\text { unit vector }
\end{aligned}
$$

$N=$ number of cells on square grid in each direction

$N_{c}=$ number of coarse grid cycles

$p=$ pressure

$p_{0}, p_{1}, p_{2}=$ pressure terms perturbation solution

$\widetilde{p}_{1}, \widetilde{p}_{2}=$ pressure terms perturbation solution

$R^{\prime}=$ surface feature function

$\bar{R}=$ relative difference Stokes-Reynolds solution

$r^{p}=L_{2}$ norm of the residue of the continuity equation

$u=$ velocity $x$-direction

$u_{c}=$ characteristic velocity $x$-direction $[\mathrm{m} / \mathrm{s}]$

$u_{0}, u_{1}, u_{2}=$ velocity terms perturbation solution

$U_{0}=$ velocity lower surface

$U_{h}=$ velocity upper surface

$x, x^{1}=$ in film coordinate

$x^{2}=$ cross-film coordinate

$w=$ velocity $z$-direction

$w_{0}, w_{1}$

$w_{2}=$ velocity terms perturbation solution

$\mathbf{x}=(x, z)=$ Cartesian coordinates

$z=$ cross-film coordinate

$\beta=$ parameter $x$-coordinate transformation

$\gamma=\epsilon^{2}=$ perturbation parameter

$\Gamma_{i}=$ domain boundary

$\Delta x=$ parameter $x$-coordinate transformation

$\epsilon=H / L$

$\eta_{c}=$ characteristic viscosity [Pas]

$\kappa_{s}=$ surface feature decay parameter

$\Lambda=$ wavelength surface feature $[\mathrm{m}]$

$\Lambda_{c}=$ characteristic wavelength

$\lambda_{s}=$ wavelength sinoidal surface feature

$\Lambda_{s}=$ width parameter surface feature

$\xi^{1}=$ computational coordinates in film

$\xi^{2}=$ computational coordinate cross-film

$\rho=$ density

$\rho_{c}=$ characteristic density $\left[\mathrm{kg} / \mathrm{m}^{3}\right]$

$\Psi=$ stream function

$\Omega=$ flow domain

\section{Relations}

$$
\begin{aligned}
\mathbf{a}^{(k)} \cdot \mathbf{a}_{(l)} & =\delta_{l}^{k} \\
\mathbf{a}^{(1)} & =(1 / \sqrt{g})\left(a_{(2)}^{2},-a_{(2)}^{1}\right)^{T} \\
\mathbf{a}^{(2)} & =(1 / \sqrt{g})\left(-a_{(1)}^{2}, a_{(1)}^{1}\right)^{T}
\end{aligned}
$$

\section{References}

[1] Reynolds, O., 1886, "On the Theory of Lubrication and Its Application to Mr. Beauchamps Tower's Experiments, Including an Experimental Determination of the Viscosity of Olive Oil," Phil. Trans. of the Royal Society, 177, pp. $157-234$.

[2] Elrod, H. G., 1979, “A General Theory for Laminar Lubrication With Reynolds Roughness,” ASME J. Lubr. Technol., 101, pp. 8-14.

[3] Tzeng, S. T., and Saibel, E., 1967, "Surface Roughness Effects on Slider Bearing Lubrication," ASLE Trans., 10, pp. 334-338.

[4] Tzeng, S. T., and Saibel, E., 1967, "On the Effects of Surface Roughness in the Hydrodynamic Lubrication Theory of a Short Journal Bearing," Wear, 10, pp. $179-184$.

[5] Christensen, H., 1970, "Stochastic Models for Hydrodynamic Lubrication of Rough Surfaces,” Proc. Inst. Mech. Eng., 184(1), pp. 1013-1026.

[6] Christensen, H., 1971, "Some Aspects of the Functional Influence of Surface Roughness in Lubrication," Wear, 17, pp. 149-163.

[7] Christensen, H., and Tonder, K., 1971, "The Hydrodynamic Lubrication of Rough Bearing Surfaces of Finite Width,” ASME J. Tribol., pp. 324-330.

[8] Christensen, H., and Tonder, K., 1973, "The Hydrodynamic Lubrication of Rough Journal Bearings,” ASME J. Tribol., pp. 166-172.

[9] Elrod, H. G., 1973, “Thin-Film Lubrication Theory for Newtonian Fluids With Surfaces Possessing Striated Roughness or Grooving," ASME J. Lubr. Technol., pp. 484-489.

[10] Elrod, H. G., 1977, “A Review of Theories for Fluid Dynamic Effects of Roughness on Laminar Lubricating Films," Proceedings of the 4th Leeds Lyon Symposium on Tribology, Mechanical Engineering Publications, pp. 11-26.

[11] Cheng, H. S., and Dyson, A., 1977, "Elastohydrodynamic Lubrication of Circumferentially-Ground Rough Disks," ASLE Trans., 21, pp. 25-40.

[12] Chow, L. S. H., and Cheng, H. S., 1976, "The Effect of Surface Roughness on the Average Film Thickness Between Lubricated Rollers," ASME J. Tribol., pp. $177-124$. 
[13] Chow, P. L., and Saibel, E. A., 1978, “On the Roughness Effect in Hydrodynamic Lubrication,” ASME J. Tribol., 100, pp. 176-180.

[14] Sun, D. C., 1978, "On the Effects of Two-Dimensional Reynolds Roughness in Hydrodynamic Lubrication," Proc. R. Soc. London, Ser. A, 364, pp. 89 106.

[15] Phan-Thien, N., 1981, "On the Effects of Parallel and Transverse Stationary Random Surface Roughness in Hydrodynamics Lubrication,” Proc. R. Soc. London, Ser. A, 374, pp. 569-591.

[16] Phan-Thien, N., 1982, "Hydrodynamic Lubrication of Rough Surfaces," Proc. R. Soc. London, Ser. A, 383, pp. 439-446.

[17] Phan-Thien, N., 1982, "On the Mean Reynolds Equation in the Presence of Homogeneous Random Surface Roughness," ASME J. Tribol., 49, pp. 476480.

[18] Phan-Thien, N., and Atkinson, J. D., 1982, “On the Effects of Homogeneous Reynolds Roughness in a Two-Dimensional Slider Bearing With Exponential Film Thickness," ASME J. Tribol., 104, pp. 220-226.

[19] Prakash, J., 1984, “On the Lubrication of Rough Rollers," ASME J. Tribol., 106, pp. 211-217.

[20] Patir, N., and Cheng, H. S., 1978, “An Average Flow Model for Determining Effects of Three-Dimensional Roughness on Partial Hydrodynamic Lubrication," ASME J. Tribol., 100, pp. 12-17.

[21] Patir, N., and Cheng, H. S., 1979, "Application of Average Flow Model to Lubrication Between Rough Sliding Surfaces," ASME J. Tribol., 101, pp. $220-230$.

[22] Teale, J. L., and Lebeck, A. O., 1980, “An Evaluation of the Average Flow Model for Surface Roughness Effects in Lubrication,” ASME J. Tribol., 106, pp. 440-447.

[23] Tripp, J. H., 1983, "Surface Roughness Effects in Hydrodynamic Lubrication: The Flow Factor Method," ASME J. Tribol., 105, pp. 458-465.

[24] Sadeghi, F., and Sui, P. C., 1989, "Compressible Elastohydrodynamic Lubrication of Rough Surfaces," ASME J. Tribol., 99, pp. 2-6.

[25] Patir, N., and Cheng, H. S., 1978, "Effect of Surface Roughness Orientation on the Central Film Thickness in EHD Contacts," Proc. 1977 Leeds Lyon Symposium on Tribology, Elsevier, pp. 15-21.

[26] Tripp, J. H., and Hamrock, B. J., 1985, "Surface Roughness Effects in Elastohydrodynamic Contacts," Proc. 1984 Leeds Lyon Symposium on Tribology, Elsevier, pp. 30-39.

[27] Goglia, P. R., Cusano, C., and Conry, T. F., 1984, “The Effects of Irregularities on the Elastohydrodynamic Lubrication of Sliding Line Contacts: Part I-Single Irregularities," ASME J. Tribol., 106, pp. 104-112.

[28] Goglia, P. R., Cusano, C., and Conry, T. F., 1984, "The Effects of Irregularities on the Elastohydrodynamic Lubrication of Sliding Line Contacts: Part IIWavy Surfaces," ASME J. Tribol., 107, pp. 113-119.

[29] Venner, C. H., Kaneta, M., and Lubrecht, A. A., 2000, "Surface Roughness in Elastohydrodynamically Lubricated Contacts," Proceedings of the 26th LeedsLyon Conference, Leeds, Sept. 1999, Elsevier Tribology Series, 38, Dowson et al., eds., pp. 25-36.

[30] Venner, C. H., Kaneta, M., Nishikawa, H., and Jacod, B., 2001, "Effects of Waviness on the Film Thickness in a Circular EHL Contact Under Rolling/ Sliding," Proceedings Int. Tribology Conference, Nagasaki 2000, Japanese Society of Tribologists, ISBN 4-9900139-4-8, pp. 631-636.

[31] Wijnant, Y. H., Venner, C. H., and Larsson, R., 1999, "Effects of Structural Vibrations on the Film Thickness in an EHL Circular Contact," ASME J. Tribol., 121, pp. 259-264.

[32] Kaneta, M., 1992, "Effects of Surface Roughness in Elastohydrodynamic Lubrication," JSME Int. J., Ser. III, 35(4), pp. 535-546.

[33] Venner, C. H., and Lubrecht, A. A., 1994, "Numerical Simulation of a Transverse Ridge in a Circular EHL Contact Under Rolling/Sliding," ASME J. Tribol., 116, pp. 751-761.

[34] Sun, D. C., and Chen, K. K., 1977, "First Effects of Stokes Roughness on Hydrodynamic Lubrication," ASME J. Lubr. Technol., pp. 2-9.

[35] Phan-Thien, N., 1981, "On the Effects of the Reynolds and Stokes Surface
Roughnesses in a Two-Dimensional Slider Bearing," Proc. R. Soc. London, Ser. A, 377, pp. 349-362.

[36] Myllerup, C. M., and Hamrock, B. J., 1992, "Local Effects in Thin Film Lubrication," Proceedings of the 19th Leeds Lyon Symposium on Tribology, D. Dowson et al., eds., Elsevier Tribology Series, 25, pp. 39-57.

[37] Myllerup, C. M., and Hamrock, B. J., 1994, "Perturbation Approach to Hydrodynamic Lubrication Theory," ASME J. Tribol., 116, pp. 110-118.

[38] Noordmans, J., "Solutions of Highly Anisotropic Stokes Equations for Lubrication Problems," Proceedings of the Second ECCOMAS Conference on Numerical Methods in Engineering, 9-13 September 1996, Paris, France, J. A. Desideri, P. Le Tallec, E. Onate, J. Periaux, E. Stein, eds., John Wiley \& Sons, Chichester, pp. 960-965.

[39] Schäfer, C. T., Giese, P., and Woolley, N. H., 1999, "Elastohydrodynamically Lubricated Line Contact Based on the Navier-Stokes Equations," Proceeding of the 26th Leeds Lyon Conference on Tribology, Elsevier Tribology Series, D. Dowson et al., eds., 38, pp. 57-69.

[40] Almqvist, T., 2001, "Numerical Simulation of Elastohydrodynamic and Hydrodynamic Lubrication Using the Navier-Stokes and Reynolds Equations," Licentiate Thesis, Luleä University of Technology, Department of Mechanical Engineering, Sweden.

[41] Bair, S., Khonsari, M., and Winer, W. O., 1998, "High-Pressure Rheology of Lubricants and Limitations of the Reynolds Equation," Tribol. Int., 31(10), pp. $573-586$.

[42] Odyck van, D. E. A., 2001, "Stokes Flow in Thin Films," Ph.D. thesis, University of Twente, The Netherlands.

[43] Odyck van, D. E. A., and Venner, C. H., 2001, "Compressible Stokes Flow in Thin Films," internal report, University of Twente, Enschede.

[44] Oosterlee, K., 1993. "Robust Multigrid Methods for the Steady and Unsteady Incompressible Navier-Stokes Equations in General Coordinates," Ph.D. thesis, Delft University, The Netherlands.

[45] Warsi, Z. U. A., 1993, Fluid Dynamics, Theoretical and Computational Approaches, CRC Press.

[46] Harlow, F., and Welch, J., 1965, "Numerical Calculation of Time-Dependent Viscous Incompressible Flow,” Phys. Fluids, 8, pp. 2182-2189.

[47] Richtmyer, R. D., and Morton, K. W., 1967, "Difference Methods for Initial Value Problems," $2^{\text {nd }}$ ed., Wiley-Interscience, London.

[48] Hackbusch, W., 1992. "Elliptic Differential Equations, Theory and Numerical Treatment," Springer Series in Computational Mathematics, 18, SpringerVerlag, Berlin.

[49] Brandt, A., 1982, "Guide to Multigrid Development," Multigrid Methods: Lecture Notes in Mathematics, 960, W. Hackbusch and U. Trottenberg, eds., Springer Verlag, Berlin, pp. 220-312.

[50] Stüben, K., and Trottenberg, U., 1982, "Multigrid Methods: Fundamental Algorithms, Model Problem Analysis and Applications," Multigrid Methods: Lecture Notes in Mathematics, 960, W. Hackbusch and U. Trottenberg, eds., Springer-Verlag, Berlin, pp. 1-176.

[51] Hackbusch, W., 1985, Multi-Grid Methods and Applications, Springer-Verlag, Berlin.

[52] Briggs, W. L., 1987, A Multigrid Tutorial, SIAM.

[53] Wesseling, P., 1992, An Introduction to Multigrid Methods, John Wiley \& Sons.

[54] Joppich, W., 1996, Grundlagen der Mehrgittermethode, GMD, Sankt Augustin.

[55] Venner, C. H., and Lubrecht, A. A., 2000, Multigrid Methods in Lubrication, Elseviers Tribology Series, 37, D. Dowson, ed., ISBN 0-444-50503-2, Elsevier, New York.

[56] Vanka, S. P., 1985, "Block-Implicit Calculation of Steady Turbulent Recirculating Flows," Int. J. Heat Mass Transf., 28, pp. 2093-2103.

[57] Moffat, H. K., 1964, "Viscous and Resistive Eddies Near a Sharp Corner," J. Fluid Mech., 18, pp. 1-18. 